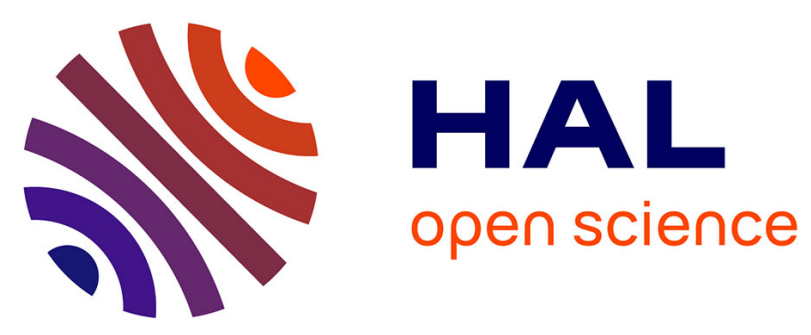

\title{
Cholangiocarcinoma stem-like subset shapes tumor-initiating niche by educating associated macrophages
}

Chiara Raggi, Margherita Correnti, Antonio Sica, Jesper B. Andersen, Vincenzo Cardinale, Domenico Alvaro, Giovanna Chiorino, Elisa Forti, Shannon S. Glaser, Gianfranco Alpini, et al.

\section{To cite this version:}

Chiara Raggi, Margherita Correnti, Antonio Sica, Jesper B. Andersen, Vincenzo Cardinale, et al.. Cholangiocarcinoma stem-like subset shapes tumor-initiating niche by educating associated macrophages. Journal of Hepatology, 2017, 66 (1), pp.102-115. 10.1016/j.jhep.2016.08.012 . hal01414693

HAL Id: hal-01414693

https://hal-univ-rennes1.archives-ouvertes.fr/hal-01414693

Submitted on 29 May 2017

HAL is a multi-disciplinary open access archive for the deposit and dissemination of scientific research documents, whether they are published or not. The documents may come from teaching and research institutions in France or abroad, or from public or private research centers.
L'archive ouverte pluridisciplinaire HAL, est destinée au dépôt et à la diffusion de documents scientifiques de niveau recherche, publiés ou non, émanant des établissements d'enseignement et de recherche français ou étrangers, des laboratoires publics ou privés. 


\section{Cholangiocarcinoma Stem-like Cells Shapes Tumor-initiating Niche by Regulating Associated Macrophages}

Chiara Raggi $1^{1 \#^{*}}$, Margherita Correnti $1^{1^{*}}$, Antonio Sica ${ }^{3,4}$, Jesper B Andersen ${ }^{5}$, Vincenzo Cardinale ${ }^{6}$, Domenico Alvaro ${ }^{6}$, Giovanna Chiorino ${ }^{7}$, Elisa Forti ${ }^{1}$, Shannon Glaser ${ }^{8}$, Gianfranco Alpini ${ }^{8}$, Annarita Destro $^{9}$, Francesca Sozio ${ }^{10,11}$, Luca Di Tommaso ${ }^{9,12}$, Massimo Roncalli ${ }^{9,12}$, Jesus M Banales ${ }^{13}$, Cédric Coulouarn ${ }^{14}$, Luis Bujanda ${ }^{13}$, Guido Torzilli ${ }^{15}$, Pietro Invernizzi ${ }^{1,2 \#}$

${ }^{1}$ Center for Autoimmune Liver Diseases, Humanitas Clinical and Research Center, Rozzano, Italy

${ }^{2}$ Program for Autoimmune Liver Diseases, International Center for Digestive Health, Department of Medicine and Surgery, University of Milan-Bicocca, Italy

${ }^{3}$ Laboratory of Molecular Immunology, Humanitas Clinical and Research Center, Rozzano, Italy

${ }^{4}$ Department of Pharmaceutical Sciences, University of Piemonte Orientale "Amedeo Avogadro" Novara, Italy

${ }^{5}$ Biotech Research and Innovation Centre, University of Copenhagen, Copenhagen, Denmark

${ }^{6}$ Department of Medico-Surgical Sciences and Biotechnologies, Sapienza University of Rome, Rome, Italy

${ }^{7}$ Cancer Genomics Laboratory, Fondazione Edo ed Elvo Tempia, Biella, Italy

${ }^{8}$ Research, Central Texas Veterans Health Care System, Scott \& White Digestive Disease Research Center, Scott \& White, Department of Medicine, Texas A\&M Health Science Center, College of Medicine, Temple, TX

${ }^{9}$ Pathology Unit, Humanitas Research Hospital, Rozzano, Italy

${ }^{10}$ Leukocyte Migration Laboratory, Humanitas Clinical and Research Center, Rozzano, Italy

${ }^{11}$ Department of Molecular and Translational Medicine, University of Brescia, Brescia, Italy

${ }^{12}$ University of Milan Medical School, Milan, Italy

${ }^{13}$ Department of Liver and Gastrointestinal Diseases, Biodonostia Research Institute, Donostia University Hospital, University of the Basque Country (UPV/EHU), CIBERehd, Ikerbasque, San Sebastián, Spain

${ }^{14}$ Inserm U991, Hôpital Pontchaillou 35033 Rennes cedex, France

${ }^{15}$ Department of Hepatobiliary and General Surgery, Humanitas Research Hospital, Rozzano, Italy

*First Authors 


\section{Contact:}

Raggi Chiara, ${ }^{1}$ Center for Autoimmune Liver Diseases, Humanitas Clinical and Research Center, via Manzoni 113, 20089 Rozzano (Milan), Italy. Email: chiara.raggi@humanitasresearch.it. Phone: +39 (02) 8224 5127;

Invernizzi Pietro, Program for Autoimmune Liver Diseases, International Center for Digestive Health, Department of Medicine and Surgery, University of Milan-Bicocca, Via Cadore 48, 20900 Monza (MB), Italy. Email: pietro.invernizzi@unimib.it. Tel +39 0264488052.

Key Words: Cholangiocarcinoma, Cancer Stem Cells, Tumor-Associated Macrophages

List of Abbreviations: Cholangiocarcinoma (CCA), Hepatocarcinoma (HCC), Cancer Stem Cells (CSCs), Tumor-Associated Macrophages (TAMs), Epithelial Mesenchymal Transition (EMT)

Words Count: 6527

\section{Number of Figure: 7}

\section{Number of Tables: 1}

Conflicts of interest: The authors disclose no conflicts.

Financial support: The study was supported partly by the Italian Foundation of Cancer Research award (MFGA17588) to Dr. Raggi, partly by the Dr. Nicholas C. Hightower Centennial Chair of Gastroenterology from Scott \& White, a VA Research Career Scientist Award, VA Merit award (5I01BX000574) to Dr. Alpini and a VA Merit Award (5I01BX002192) to Dr. Glaser. Dr. Raggi was supported by U. Veronesi Foundation Post-Doctoral fellowship.

Author' contribution: Study design: CR; Data acquisition/analysis: MC JBA AD FS LDT EF; Manuscript drafting: CR; Critical revision: AS GA DA; Statistical analysis: GC; Obtained funding: CR GA PI; Technical support: VC SG MR JMB CC GT CC JMB LB 


\section{ABSTRACT}

\section{Word count 293}

Background and Aims: Therapeutically challenging subset, termed cancer stem cells (CSCs) are responsible for cholangiocarcinoma (CCA) clinical severity. Presence of tumorassociated macrophages (TAMs) has prognostic significance in CCA and other malignancies. Thus, we hypothesized that CSCs may actively shape their tumor-supportive immune niche. Methods: CCA cells were cultured in 3D-condition to generate spheres (SPH). CCA-SPH analysis of in vivo tumorigenic-engraftment in immune-deficient mice and molecular characterization was performed. In vitro and in vivo effect of CCA-SPH on macrophageprecursors was tested after culturing healthy donor CD14+ with CCA-SPH conditioned medium .

Results: CCA-SPHs engrafted $100 \%$ of transplanted mice, revealed a significant 20.3 -fold increase in tumor-initiating fraction $(p=0.0011)$ and a sustained tumorigenic potential through diverse xenograft-generations. Moreover, CCA-SPHs were highly enriched for CSC, liver cancer and embryonic stem cell markers both at gene and protein levels. Next, FACSanalysis showed that in presence of CCA-SPH-CM, CD14+ expressed key macrophage (MØ) markers (CD68, CD115, HLA-DR, CD206) indicating that CCA-SPH- conditioned medium was a strong MØ-activator. Gene expression profile of CCA-SPH activated MØ (SPH MØ) revealed unique molecular TAM-like features confirmed by high invasion capacity. Also, freshly isolated MØs from CCA-resections recapitulated similar molecular phenotype of in vitro educated-MØs. Consistently with invasive features, largest CD163+ set was found in tumor-front of human CCA specimens ( $n=23)$ and correlated with high level of serum CA19.9 ( $n=17)$. Among mediators released by CCA-SPHs, only IL13, IL34 and osteoactivin were detected and further confirmed in CCA patient sera $(n=12)$. Surprisingly, significant 
association of IL13, IL34 and osteoactivin with SPH stem-like genes was provided by CCA database $(n=104)$. In vitro combination of IL13, IL34, osteoactivin was responsible for MØdifferentiation and invasion as well as for in vivo tumor-promoting effect.

Conclusion: CCA-CSCs molded a specific subset of stem-like associated-MØs thus providing a rationale for a synergistic therapeutic strategy for CCA-disease.

\section{LAY SUMMARY}

Immune plasticity represents an important hallmark of tumor outcome. Since cancer stem cells are able to manipulate stromal cells to their needs, a better definition of key deregulated immune subtype responsible to cooperate in supporting tumor initiation may facilitate the development of new therapeutic approaches. Considering that human cholangiocarcinoma represents a clinical emergency, it is essential to move to predictive models to understand the adaptive process of macrophage component (imprinting, polarization and maintenance) engaged by tumor stem-like compartment.

\section{INTRODUCTION}

Neoplastic transformation of intra- and extra-hepatic biliary epithelial cells (cholangiocytes) gives rise to cholangiocarcinoma (CCA), a major form of primary liver cancer along with hepatocellular carcinoma (HCC). Due to its aggressiveness, late diagnosis and treatmentrefractory nature, CCA represents a clinical emergency [1, 2]. CCA incidence and mortality rates are increasing worldwide and therapeutic options are limited.

Due to its desmoplastic nature, CCA is associated with massive presence of stromal cells [3, 4]. Among various immune-subgroups (e.g., T cells, neutrophils, granulocytes) [5-8], CCA- 
associated macrophages represent a poor defined but very intriguing immune-subset. Indeed, high tumor-associated macrophage (TAM) density significantly correlated with poor prognosis, unfavorable survival and metastasis tendency in CCA patients, suggesting a major role of macrophages in cholangiocarcinoma progression (Reviewed in [4]).

Recently, cancer stem cells (CSCs) have been proposed as a driving force of tumor initiation, dissemination and drug-resistance thus representing a primary therapeutic target. Importantly, the view that CCA is traditionally considered to originate either from cholangiocytes or progenitor cells, has been recently challenged. Indeed, it is proven that CCA can also originate from mature hepatocytes in mouse models [9].

Although it has been shown that HCC progression is driven by CSCs [10,11], identification of CCA stem-subset is still largely unexplored and limited to classical antigenic approaches (Reviewed in [4]). Similar to normal stem cells, CSCs are believed to reside in specialized microenvironment ("CSC-niche") within tumor-context [12-14] that supports self-renewal and drug-resistance [15-19].

Tumor-stroma co-evolves together with cancer cells and plays a critical part in both malignancy initiation as well as key steps of growth and metastasis [20]. Since macrophages show a remarkable degree of plasticity during tumor development, we assumed that, depending on CSC tumorigenic-state, CSCs might actively release soluble mediators that engaged circulating-monocytes to tumor-initiating niche and prompted macrophage differentiation towards an exclusive subset of TAMs, the CSC-associated TAMs. Although 
bulk tumor cells support TAMs recruitment and activation, specific effects of CSCs on TAMs phenotype are still unexplored in human CCA.

The current study sought to 1) identify a functional CSC-subset in human CCA and 2) verify the existence of a peculiar TAM-compartment as result of a bioactive CCA stem-like cells.

\section{RESULTS}

\section{Human CCA-Spheres Retain Stem-Like Tumor-Initiating Features}

CCA-stem like cells were identified by using a functional tool of 3D culture system based on defined serum-free medium, as shown in many patient-derived tumors [21, 22]. First, established (SG231, HUCCT1, CCLP1) [23-25] and primary human intrahepatic CCA-derived (CCA4) cell lines [26] were tested for sphere-forming efficiency (SFE) as a representation of tumor stem-like subset. To this end, CCA FACS-sorted single cells were cultured in 3D nonadherent condition in a 96-well plate. Importantly, single-cell sorting prevents formation of cell-aggregation and avoids large fraction of non-stem cells to form clusters. As expected, only malignant cholangiocytes retained sphere-forming potential in contrast to normal counterparts such as normal immortalized cholangiocytes (H69) and human intrahepatic biliary epithelial cells (HiBECs) (Figure1A). However, CCA-spheres (SPH) revealed a high grade of heterogeneity in terms of efficiency (HUCCT1 and CCLP1, approximately 20\%; SG231 and CCA4, 10\%), size and morphology (FigureS1A).

Next, an extensive characterization, including evaluation of in vivo tumorigenicity in immunedeficient NOD-SCID/IL2Rg ${ }^{\text {null }}$ (NSG) mice, drug-responsiveness and gene expression analysis of CCA-SPH, was performed.

To assess CCA-SPH ability to generate tumors, spheres formed by HUCCT1, SG231, CCLP1 and CCA4 were dissociated and 1,000 cells injected subcutaneously (s.c.) into NSG mice 
(Figure1B-D) [27]. Consistently with their in vitro SFE (Figure1A), CCA-SPHs were highly tumorigenic and engrafted $100 \%$ of transplanted mice in all examined cell lines albeit with differences in tumor size (Figure1B-D). In contrast to CCAs grown in monolayer condition (MON), including H69-MON, CCA-SPHs were responsible for early tumor initiation and formation (3 weeks for HUCCT1, CCLP1; 5 weeks for SG231; 6 weeks for CCA4) during 13 weeks of observation. In addition, SPH-derived tumors (SPH-T) showed a large difference in terms of tumor weight and size compared to CCA-MON derived tumors (MON-T) (Figure1D), indicating that SPH-T retained high tumorigenic potential when xenotransplanted in animal hosts and represent an important source of de novo tumor growth. The most common method to determine frequency of self-renewing cells within tumors is a limiting-dilution cell transplantation assay. Thus, due to slight differences in tumor potential between SPH and MON in CCLP1, we specifically performed limiting-dilution transplantation assay in NSG mice. After s.c. injection of 1,000, 100 or 10 cells of both CCLP1-MON and SPH conditions, data analysis using ELDA software $[28,29]$ revealed a significant 20.3 -fold increase in the absolute number of tumor-initiating fraction (TIF) within CCLP1-SPHs (TIF=1/25) compared to MONs $(\mathrm{TIF}=1 / 505)(\mathrm{p}=0.0011$, Figure1E). Indeed, as few as $10 \mathrm{SPH}$ cells produced tumors, whereas 1,000 MON cells gave rise to fewer tumors at 13 weeks (Figure1E) confirming that SPHs were remarkably enriched in self-renewing CCA-propagating cells. Additionally, since selfrenewing stem-like cancer cells have an unlimited ability to promote tumor growth, capability for serial transplantation was also tested (Figure1F). Cells were re-isolated from CCLP1-T established from both $1000 \mathrm{SPH}$ and MON cells, propagated in short-term cultures and retransplanted into secondary recipient mice. Notably, SPHs not only sustained tumorigenic potential in serial transplantations but also progressively improved in tumor frequency. Conversely, corresponding MONs showed either dramatic increase in tumor latency and 
decrease in tumor incidence at later generations (Figure1F). Together these data indicated that, in accordance with long-term self-renewal potential, SPHs significantly maintain tumorigenic potential through diverse xenograft-generations while MONs reduced it.

Furthermore, because drug-resistance represents a key feature of cancer stem/initiating cells, 3D cultures were tested for susceptibility to chemotherapeutic agents. Indeed, in presence of common anti-CCA drugs such as cisplatin, 5-fluorouracil, oxaliplatin and gemcitabine, CCA$\mathrm{SPH}$ revealed an better survival rate compared to parental CCA-MONs, as measured by proliferation MTT assays and indicated by the $50 \%$ inhibitory concentration values (IC50) (FigureS1B). Although a remarkable heterogeneity in terms of drug-sensitivity, SPHresistance was a common phenomenon observed across all CCA lines.

Next, to corroborate our in vivo and in vitro findings, an extensive molecular characterization of CCA-SPH was achieved by PCR-arrays specific for CSC, liver cancer and embryonic stem cell (ESC) pathways. In accordance with the observed heterogeneity in SFE, tumor potential and drug-resistance, clustering analysis of differentially expressed genes for each pathway revealed SPH gene-enrichment as compared to respective MONs, specific for each cell line (Figure2A-C, FigureS2A). Indeed, CCLP1, HUCCT1 and CCA4 SPHs presented augmented expression for CSC- (55-69\%) and liver cancer-related (61-77\%) genes, whereas SG231$\mathrm{SPH}$ retained the highest fraction of ESC-related (37\%) genes (FigureS2A). However, a core of 30 common deregulated genes in all CCA-SPHs was identified. Specifically, expression of 23 CSC-genes, including key molecules for pluripotency and self-renewal (SOX2, POU5F1, KLF4, BMI1, NOTCH1, MYC, KITLG, LIN28A, MAML1, YAP1), drug-resistance and survival (ABCG2, LATS1, NFKB1, NOS2), metastasis (TGFBR1, BPM7, FGFR2) and stem-like surface markers (CD24, CD44, PROM1, THY1, EPCAM), resulted enhanced. Moreover, this 
common gene-set revealed overexpression of 6 hepatic oncogenic drivers (CDKN1A, BCL2L1, CTNNB1, IGF2, ITGB1, LEF1) and key ESC factors such as HNF4 (Figure2B), in addition to the well-described liver CSC markers CD13 and LGR5 (not included in the arrays) (Figure2C). Accordantly, further confirmation of stem-cell content was considered at protein level by FACS-analysis. Indeed, superior expression of CD13, CD44, THY1 and EPCAM were verified in CCA-SPHs from four cell lines (Figure2D, FigureS2B) thus suggesting that 3D cultures were effectively enriched for tumorigenic cells endowed with stem-like molecular traits.

Particularly, presence of the most significant CSC surface markers, such as CD44, PROM1, THY1 and EPCAM was validated by CCA transcriptome of 104 patient database [1] (FigureS2C).

Whereas gene expression profile was consistent with stem-like traits of SPH-condition, we also tested the strength of 3D culture system compared to antigenic approach. To this end, primary CCA4 was immune-sorted for standard stem-related marker THY1 and verified for in vitro sphere-ability. However, no significant differences in in vitro self-renewing capability were found between $\mathrm{THY}+$ and parental cells as control (FigureS2D, left). Next, we combined expression of stem-like markers to sphere-forming capacity, thus THY+ cells were grown in 3D condition $(\mathrm{THY}+\mathrm{SPH})$ and tested for tumorigenic potential. Notably, in vivo analysis revealed similar TIF subset between $\mathrm{THY}+\mathrm{SPH}(\mathrm{TIF}=1 / 47.8)$ and parental SPH $(\mathrm{TIF}=1 / 35.7)$ ( $p=0.2028$ FigureS2D, right) also confirmed by expression of liver cancer, CSC and ESC-like markers (FigureS2D, down). These results indicated that 3D culture system potentially represents a reliable tool and valid alternative to the antigenic approach for CCA stem-like cell selection. 
Altogether, this broad functional and molecular characterization proposed the existence of a stem/progenitor-like compartment in human CCA identified by 3D culture system. Indeed, CCA-SPHs exhibited typical stemness hallmarks, such as up-regulated stem-cell biomarkers, drug-resistance and enhanced capability to initiate malignancy.

\section{CCA Stem-Like Compartment Educates Macrophage Precursors Toward Acquisition of CSC-Associated TAM Phenotype}

In addition to diverse cellular components and ECM, CSC-milieu is enriched with tumor cell products such as growth factors, cytokines, prostaglandins and diverse protein factors [30]. Thus, to mimic a bioactive CSC-niche, freshly isolated healthy donor circulating monocytes (CD14+) were exposed to CCA-SPH conditioned medium (SPH-CM) and tested for 1) chemotactic recruitment based on migration properties and 2) macrophage (MØ) differentiation by analysis of marker expression and functional properties (Figure3, FigureS3A). First, by using a chemotaxis chamber, a higher number of CD14+ migrating towards SPH-CM gradient than MON-CM was recorded within 6h, suggesting that SPH-CM acted as a strong monocyte attractor (Figure3A). Hence, greater amount of recruited mononuclear cells by CCA-SPHs maybe find a potential explanation in creating a tumorigenic-niche supporting CSC-maintenance.

It is widely accepted that $\mathrm{M} \varnothing \mathbf{s}$ constitute an extremely heterogeneous population. Most MØs originate from blood monocytes, which differentiate into distinct $M \varnothing$ types, schematically identified as M1 (or classically activated) and M2 (or alternatively activated). It is now generally recognized that TAMs show an M2-like cancer-promoting phenotype [31-33].

To determine the effect of the CCA stem-like component on MØ-precursors, healthy donors 
CD14+ were cultured in presence of CCA SPH-CM, and after 6 days, investigated for MØdifferentiation and polarization. Consistently with observed changes in morphology (data not shown), by using a panel of conventional markers typically used for M0 classical differentiation (CD115, macrophage colony-stimulating factor receptor or M-CSF receptor), M1 (HLA-DR, human leukocyte antigen and MHC class II) and M2-TAM (CD206, macrophage mannose receptor) polarization (FigureS3B), FACS-analysis revealed that in presence of CCA SPH-CM, in vitro-educated CD14+ expressed higher CD115 level in contrast to CD14+ exposed to MON-CM or M-CSF, which acted as control for M0 classical differentiation (Figure3B, TableS1). As expected and regardless culture conditions, CCA-CM induced M $\varnothing$ differentiation of human monocytes as indicated by CD14+ CD68+ cells in both MON and SPH-CM (FigureS3C-D). Notably, more than 94\% of SPH-educated CD14+ expressed CD14, CD68 and CD115 markers (Figure3B, FigureS3C-D). Moreover, differentiation towards neutrophil lineage and dendritic cells was excluded by CD66b and CD1a staining [34, 35], also confirmed in M0-MØs (FigureS3E-F). Furthermore, CCA-SPH activated-M $\varnothing$ (SPH MØ) expressed greater levels of CD206 and HLA-DR TAM-like markers in contrast to CCA-MON activated-M $\varnothing(\mathrm{MON} M \varnothing)$ (Figure3B, TableS1). All these data indicated that both MON- and SPH-CM induced M $\varnothing$-differentiation, but SPH-CM was responsible for M $\varnothing$-acquisition of a specific CSC-dependent phenotype.

Likewise, in in vitro-educated MØs, expression genes belonging to M1 (e.g., CD80, CCL5, CXCL9, CXCL10), M2 (ALOX15, CCL18, and CCL17) and TAM-like (CD163) categories, including genes involved in ECM-remodeling and adhesion such as osteoactivin (OA), fibronectin $(F M)$, osteopontin $(O P N)$, metalloproteinase ADAM (AD10, $A D 17)$, and matrix metalloproteinase-2 (MMP2), were determined (Figure3C). Notably, SPH MØs retained 
unique molecular features with concomitant expression of TAM-like (e.g., CD163), M1-like (e.g., CCL5, CXCL9, CXCL10), M2-like (e.g., CCL17, CCL18) and matrix remodeling-related (e.g., $O A, A D 17$ and MMP2) genes (Figure3C).

Consistent with our gene expression data, SPH MØs possessed greater adhesion ability on FN-support (Figure3D) and better invasion capacity as shown by in vitro Matrigel-coated transwell assays (Figure3E) [36] compared to MON MØs. Both these features are likely associated with dynamic properties of TAM-like cells within tumor-initiating niche because of their incessant and deregulated activity regarding ECM-reorganization and turnover. Owing to their potential ability to remodel tumor-stroma, SPH MØs may invade and at the same time adhere to the ECM-component, thus providing sustenance to tumor-environment. This result showed concordance with the evidence that mononuclear phagocytes support stem-cell functions, thus contributing to tissue repair and remodeling [37].

\section{CCA humanized-mice recapitulate in vitro educated-MØ traits}

To translate our in vitro data in a human-like setting, we analyzed the in vivo immunological response of human mononuclear cells to CCA stem-like component at tumor site. Thus, a cotransplantation of human peripheral blood mononuclear cells (hPBMC) and patient derived CCA4-SPHs into NSG mice was performed (Figure4A-B, FigureS4A). As already extensively demonstrated, NSG mice bearing a targeted mutation in IL2ry gene (responsible for a severe defective murine immune component) support considerably high levels of human hematopoietic and lymphoid cell engraftment providing an exciting substitute for human immunobiology studies [16]. Therefore, CCA4-SPH-T bearing mice (when tumor size reached $50 \mathrm{~mm}^{3}, 51$ days after s.c. injection) were engrafted with three successive doses of hPBMC $\left(15 \times 10^{6} /\right.$ dose) intravenously (i.v) in the retro-orbital sinus. Efficiency of human engraftment 
was tested 6 days after first hPBMC dose and presence of human circulating mononuclear cells (mCD45-hCD45+hCD14+) was confirmed in mouse peripheral blood by FACS-analysis. In agreement with published data [16], frequency of mCD45-hCD45+hCD14+ circulating cells were almost $0,7 \%$ indicating an effective human cell-engraftment (FigureS4B-C).

At the end of experiment, both CCA4-SPH-T and CCA4-MON-T were removed and FACSanalyzed for human mononuclear cells presence. Well-matched with in vitro data, CCA4$\mathrm{SPH}-\mathrm{T}$ retained higher number of human CD45+CD68+ $(83.55 \%)$ in comparison with CCA4MON-T (70\%) (Figure4A). Again, infiltrated human CD45+CD14+ in CCA4-SPH-T displayed enhanced expression of M1-like (e.g., CD80, CXCL9), M2-like (e.g., CCL18, CCL17) and matrix remodeling-related genes (e.g., OPN, $A D 10, A D 17, M M P 2$, full-length $F N$ and oncofetal FN (ED-B)) (Figure4B).

Collectively, our in vitro and in vivo observations indicated that SPHs recruit higher amount of mononuclear cells at tumor site and CCA-SPH MØs retain a unique phenotype compared to MON MØs.

\section{Mixed phenotype of infiltrating-MØs in CCA patients}

Since a broad analysis of human CCA-TAMs has not been provided yet, we corroborated our in vitro data with a global investigation of CCA patient-associated MØs. Abundance, phenotypes and distribution of $\mathrm{M} \varnothing$ s residing in CCA intratumoral and peritumoral tissue were evaluated via immunohistochemistry (IHC). Paraffin-embedded tissue samples from 23 CCA patients were tested for tumor-promoting MØs (CD163) presence. An immunohistochemical analysis of tumor sections showed that CD163+cells were more highly expressed in tumor than peripheral tumor region (Figure5A upper, TableS2). Remarkably, analysis of M $\varnothing$ - 
distribution within tumor lesion revealed that the largest proportion of CD163+ was located in the tumor front (Figure5A, lower). On further evaluation of relationships between TAM-marker density and clinical pathological features, we found that CD163 progressively increased along with tumor grade (G2/G3>>G1) and was significantly associated with CCA pathological grade $(p=0.006$,) (Figure5B, TableS2) as well as CA19.9 serum marker (Figure5C).

Likewise, FACS-profiling of mononuclear subsets from fresh resected CCA samples confirmed CD115, CD206 as well as HLA-DR expression of infiltrating CD68+ cells (Figure5D, FigureS5A-D). In addition, isolated CD14+ cells from fresh CCA tissue [16] revealed expression of $\mathrm{M} 1$-like (e.g., CD80, CXCL9) and M2-like (e.g., CCL18) markers as well as genes involved in ECM remodeling including $O A$, FN and ED-B (Figure5E).

Similarly, transcriptome of laser capture microdissected epithelium (EPI) and stroma (S) from 23 CCA patients revealed a significant deregulation of CD115, HLA-DR, CXCL9, MMP2 and CD206 in CCA-S compared to CCA-EPI compartment (Figure5F, FigureS5E). Further validation was provided by tumor (T) transcriptome versus surrounding liver tissue (SL) in an independent data set of CCA patients ( $n=104)$ (FigureS5F).

Furthermore, $\underline{\mathrm{IHC}}$ analysis of CCA sections revealed a co-localization of both CD163 positive elements together with expression of CSC-related markers (CD44 or EPCAM) strongly supporting the potential relationship of infiltrating TAMs with CSC-niche (Figure5G).

Therefore, our comprehensive characterization showed that phenotype of CCA patient MØs retained mixed M1-M2 features, further strengthening our in vitro results.

\section{CCA-SPH Secretory Profiling Specifically Involved in MØ-Differentiation}

Intriguingly, $\underline{\mathrm{IHC}}$ data revealed that $\mathrm{SPH}$ derived tumors retained a prominent and welldefined stromal compartment attested by a-SMA staining, massive collagen presence (Red 
Sirius) and abundance of tumor-associated CD31-positive vessels as well murine F4/80 tumor-macrophages (Figure6A). This evidence suggested that SPH-cells acted differentially in creating their associated surrounding microenvironment.

Furthermore, we tested the diverse functionality of CCA cells on the surrounding niche, in particular lymphocytes as well as endothelial cells. Thus, CellTrace CFSE labeled CD4 peripheral blood lymphocytes $(\mathrm{CD} 4+\mathrm{T})$ were cultured in presence of both MON and SPH-CM. It was found that SPH-CM were not able to induce CD4+ T cell proliferation, unlike MON-CM did (Figure6B) suggesting a more immunosuppressive properties of SPH-associated microenvironment [38, 39].

Since angiogenesis is important hallmark of tumor progression, we evaluated the biological activity of CCA-CM on the induction of in vitro capillary-like structures [40]. By using the wellknown matrigel angiogenesis assay, the effects of SPH-CM and MON-CM on HUVEC cells in term of capillary tube-like formation were examined in vitro. In accordance with $\underline{\mathrm{IHC}}$ data of SPH tumor-derived tissues (Figure 6A), HUVEC cells treated with SPH-CM for 24h revealed the highest neovascular response in comparison to $\mathrm{MON}-\mathrm{CM}$ treatment (Figure $6 \mathrm{C}$, Figure S6A).

Hence, based on this result, we investigated the presence of specific soluble mediators released by SPHs using enzyme-linked immunosorbent assay (ELISA) profiling of conditioned media. Among 37 tested molecules (chemokines, cytokines, interleukins) specifically involved in $M \varnothing$-differentiation, activation and recruitment, several factors were secreted at extremely low levels $(<50 \mathrm{pg} / \mathrm{mL})$, whereas 16 showed significant amount in both CCA MON and SPHCM (Figure6D, TableS3). A 3-level analysis identified 1) common SPH and MON, 2) MONspecific, 3) SPH-specific released factors. First, we found that both SPH and MON produced 
high VEGF concentrations. Therefore, VEGF role in driving $M \varnothing$-differentiation was in vitro investigated. For that reason, healthy donor CD14+ were cultured for 6 days in presence of VEGF added to fresh monocyte medium, not tumor conditioned (FigureS6B). Clearly, FACSprofile reveals enriched expression of CD68 and CD115 as classical MØ markers (FigureS6B) suggesting its contribution in CCA MØ-differentiation instead of classically described M-CSF. Notably, both SPH and MON MØs overexpressed corresponding VEGF receptors (VEGFR1-3) (FigureS6C).

Nevertheless, a cell line-specific MON secretory profile was identified, with altered supernatant levels of GM-CSF, M-CSF, TNFa, TGF $\beta$ (HUCCT1), GM-CSF, CCL22, CCL20, CXCL8 (SG231), CCL2, and CXCL18 (CCLP1 cells), CXCL11 (CCA4) (TableS3). However, release of soluble mediators was cell line-dependent and no common molecules among CCAMONs were recognized.

Crucially, a specific secretory SPH-profile consisting of IL13, IL34 and OA was identified in all tested cell lines (Figure6D, FigureS6D) and more importantly SPH MØs overexpressed receptors for IL13 (IL13Ra1, IL13Ra2, IL4R), OA (CD44, ITGA5, ITGB3, SDC1, SDC4) (Figure6E) as well as for IL34 (CD115) already shown in Figure3B. Therefore, these data proposed the potential implication of IL13, OA and IL34 in the MØ-acquisition of CSC-specific phenotype.

Surprisingly, elevated levels of circulating IL13, OA and IL34 were significantly found in CCA patient group $(n=12)$ compared to healthy control subjects $(n=12)$, proposing a potential association with CCA-disease (Figure6F, TableS4). However, due to limited availability of human CCA specimens, important question such correlation between serum level and abundance of TAMs as well clinical pathological features of CCA patients was not addressed. Nonetheless, correlative data were provided by a well-described transcriptome database of 
104 CCA patients [1]. Amazingly, Pearson correlation coefficient test showed significant associations of specific CCA-SPH released molecules (IL13, OA, IL34) with SPH stem-like genes such as BMI1, CTNNB1, CD44, KITGL, KLF4, LEF1, LIN28A, MAML1, POUF5, SOX2

and THY1 (Table 1) reinforcing the importance of CCA stem-secretory profiling in tumor context in CCA patients. Furthermore, intimate connection between CSCs and tumor-MØs in human CCA was also denoted by meaningful correlations between stem-like SPH and SPH MØ-molecular traits (TableS5).

\section{IL13, IL34, and OA Are Required for Acquisition of CSC-like TAM Identity}

To confirm the contribution of IL13, OA, IL34 in shaping molecular and functional aspect of SPH MØs, inhibition of these molecules by addition of single or combination neutralizing antibodies to SPH-CM (previously in vitro tested, to define an effective and non toxic concentration, Figure7,FigureS7A), was tested in vitro and in vivo. Strikingly, our results indicated a down-regulation of CD115, CD206 and HLA-DR proteins (Figure7A, TableS6) as well as gene expression (CXCL9, CXCL10, CCL18, CCL5, CD163, OPN and AD17) (Figure7B) in addition to loss of functional properties such as migration, M $\varnothing$-invasion and adhesion (Figure7C) suggesting a shift to MON MØ-phenotype. Particularly, each single IL13, IL34, or OA seemed to be independently involved in CD115 (Figure7A). More importantly, only OA molecule is accountable for CD206 and HLA-DR expression (Figure7A) as well as MØ-invasion and adhesion (Figure7C), whereas combination of neutralizing antibody has a major impact in diminishing the expression of M1, M2 and ECM remodeling genes (Figure7B). Well-matched with SPH-CM inhibition, the addition of these molecules to MON-CM reproduced same SPH MØ differentiative impact. Indeed, IL13, IL34, and OA were able to restore SPH MØ-like profile with overexpression of CD115, HLA-DR and CD206 markers and 
presence of an equal amount of CD68 protein (Figure7A, FigureS7B-C, TableS6). Remarkably, our data displayed the re-expression of specific gene-set (CXCL9, CXCL10, CCL18, CCL5, CD163, OPN and AD17) (Figure7B, FigureS7D) as well as re-acquisition of functional properties such as monocyte recruitment, M $\varnothing$-invasion and adhesion (Figure7C, FigureS7E-F). Pertinently, all these results pointed to a peculiar contribution of these molecules in driving SPH MØ-differentiation. Thus, we concluded that although the underlying mechanism need to be explored, combination of IL13, OA, IL34 directly trigger differentiation of monocytes into stem-like TAM-subtype.

Furthermore, to investigate the significance of diverse M $\varnothing$-subsets in supporting tumorigenicity, three diverse in vitro-educated $\mathrm{M} \varnothing$-subsets such as 1) SPH MØ, 2) MON MØ, 3) $\mathrm{MON}(+\mathrm{IL} 13+\mathrm{OA}+\mathrm{IL} 34) \mathrm{M} \varnothing, 4) \mathrm{SPH}$ (+single or combination of antibodies against IL13, OA, IL34) MØ were co-injected with CCA-MONs into NSG mice. As expected, MON MØs enhanced tumor growth of MON cells injected alone as already described in diverse study for TAMs. Outstandingly, SPH MØs boosted tumorigenic potential compared to MON MØs, indicating that CSC-associated TAMs increased in vivo tumor-promoting effect of CCA-CSCs. As confirmation, co-injected MON with (+IL13+OA+IL34)M Øs reproduced similar SPH M tumorigenic effect (Figure7D), proving direct functional relationship between CSC TAMs and tumor outcome. 


\section{DISCUSSION}

Current CCA treatment strategies are largely ineffective. Therefore, understanding the pathogenesis of this disease is essential for identifying potential curative targets. Concept of stemness-driven carcinogenesis highlights the existence of a therapeutically challenging subset responsible for tumor initiation, dissemination and drug-resistance termed CSCs. Our study mainly focused on exploration of stem-compartment in human intrahepatic CCA by using a 3D culture system as a well-known functional tool to select and enriched for tumorstem like cells. Indeed the absence of CCA-CSC specific markers limits the use of classical antigenic approach. Thus, by using in vitro spherogenicity and in vivo tumorigenicity as criteria of stemness properties, for the first time we provided extensive molecular and functional evidence for CCA stem-like compartment identified by 3D cultures.

Secondly, here we proposed that tumor stem-like cells might model their immunopathologicalniche according to their necessities. Indeed, we focused on CSC-secretome responsible for infiltrating-monocyte recruitment and MØ-priming. Pertinently, aim of this study was the exploration of CSC impact on TAM traits in CCA. Indeed, we showed that TAMs acquire a peculiar phenotype dependent on bioactive CSC-secretome. Further studies are required to deepen SPH MØ properties on tumorigenic potential and drug-resistance of SPH cells.

A variety of modulators released by stromal and tumor cells recruit circulating monocytes to tumor sites, cause their differentiation into MØs and severely affect their functions [20, 41-43]. Our results showed profound molecular and functional differences among MØ-activated by CCA-SPH in comparison to MON MØs suggesting that different TAM-compartments within same tumor may reflect diverse responses to divergent local signals. Indeed, tumor stem-like associated-TAMs displayed mixed M1-M2 molecular traits including high invasion and 
adhesion capability. Hence, a mixed state of $M \varnothing$-subsets reinforced the concept of TAM plasticity. It is important to note that CSC ability to modulate a macrophage dominated inflammatory response is similar to the reactive phenotype displayed by cholangiocytes of dysplastic bile ducts in a mouse model of congenital hepatic fibrosis [44], a disease with high risk of CCA development.

Of relevance, our findings indicated that CCA-SPH released molecules, such as IL13, OA and IL34, model the macrophage component associated to CSCs. Interestingly, IL34 has recently been identified as a second ligand for hematopoietic colony stimulating factor-1 receptor (CSF-1R) together with the cytokine CSF1, also known as M-CSF [45]. CSF1-R activation establishes signaling cascades leading to differentiation and functionality of monocytes, tissue-M $\varnothing$ s and antigen-presenting dendritic cells [31, 41]. Thus, M $\varnothing$-differentiation may be possibly driven by IL34 or alternative mechanisms (e.g. VEGF) proposing new clues for CCAimmunotherapy strategies [42].

Consistently, IL13 has been described as a typical $\mathrm{Th}_{2}$ type cytokine that, together with IL4, generated alternatively activated M2-MØs [31, 41]. This clearly suggested that SPH inducedMØs resemble M2-polarized cells.

Lastly, OA (also known as glycoprotein non-metastatic melanoma protein b [gpnmb]), a type 1 transmembrane glycoprotein, is produced by embryonic nervous system, developing nephrons, osteoblasts and osteoclasts. Remarkably, OA is overexpressed in patients with glioblastoma multiform and significantly correlated with poor outcome [46]. Moreover, in 
breast cancer cells, OA high expression levels are required for their enhanced invasiveness and osteolytic bone metastases formation[47].

Together our observations pointed to a stem-like secretome (IL13, OA, IL34 production) and resulting-M $\varnothing$ phenotype in human $\mathrm{CCA}$. Although, additional studies are warranted to formally dissect molecular mechanisms underlying differentiation of recruited monocytes towards SPH MØ-subset, we provide a rationale for IL13, OA and IL34 targeting. Indeed, extra analyses are essential in demonstrating the base knowledge for effective prevention and/or treatment of malignant SPH MØ-subset, thus leading to exploration of combination therapies in CCA patients.

Notably, despite several limitations, our humanized xenograft (s.c. patient CCA-SPH and i.v. hPBMC) highlights SPH biological relevance in priming associated-MØs. In this respect, we knowledge that orthotopic allograft models for human CCA represent the impeccable tool to recapitulate key clinical, cellular and molecular features of this aggressive tumor [48]. While diverse animal models of CCA (including numerous new genetic models) do exist, an effective patient-like CCA animal model remains to be established [48]. Thus, this lack represents a limitation in the understanding the CCA pathogenesis. Nevertheless, we presented a human CCA-like setting as preclinical platform to investigate mechanisms bridging CCA-disease to CCA-infiltrating MØs for synergistic therapies. To this end, in the contest of nanotechnology medicine, in vivo administration of IL13, OA and IL34 antibodies combined with chemotherapies, potentially represent a versatile biomedical tool to improve CCA outcome.

\section{FIGURE LEGENDS}


Fig. 1. CCA self-renewal capacity in vitro and tumorigenic potential in vivo.

A) Sphere-forming efficiency (SFE) of CCAs (HUCCT1, SG231, CCLP1, CCA4) and normal cholangiocytes (HiBECs,H69) calculated by dividing the sphere number by number of single cells seeded and expressed as a percentage. Mean \pm SEM ( $n=4, p$ value versus H69 and HiBECS by Student $t$ test, ${ }^{* * *} p \leq 0.001$ ).

Sphere tumorigenic capacity in NSG mice: B) Tumor growth kinetic $(n=5)$, C) frequency and D) weight of generated tumors at 13weeks after subcutaneous injection into NSG mice of $1,000 \mathrm{SPH} / \mathrm{MON}$ isolated cells as monitored by weekly palpation. Mean \pm SEM ( $p$ value versus MON-T by Student $t$ test).

E) Self-renewing CCLP1 cells calculated by ELDA program (see Methods section) ( $p$ value versus MON-tumor initiating fraction (TIF) by Student $t$ test).

F) Serial transplantations of 1,000 CCLP1 SPH/MON cells into flanks of NSG mice $(n=4)$.

Fig. 2. Molecular properties of CCA spheres.

A) Pathway-focused qRT-PCR arrays. Hierarchical clustering distinguished each CCA-SPH type based on significant gene expression compared to CCA-MON. Data first centered and normalized, then clustered using centered correlation metrics with complete linkage. Dendrograms depict similarity of individual genes and cases. Right side figure indicates clusters of coordinately expressed genes with higher expression levels in CCA-SPH than CCA-MON. Relative gene expression level depicted according to the scale bar.

B) List of commonly up-regulated genes in SPH of all CCA cell lines. Genes divided according to pathway of belonging.

C) Relative expression of CD13 and LGR5 transcript-encoding markers. GAPDH as internal control. All mRNA levels are presented as fold changes normalized to 1 (mean expression of monolayer). Mean $\pm \operatorname{SEM}\left(n=3, p\right.$ value versus MON by Student $t$ test, ${ }^{*} p \leq 0.05,{ }^{* *} p \leq 0.01,{ }^{* *}$ $\mathrm{p} \leq 0.001)$.

D) FACS-profile of CD44, CD13, THY, EPCAM.

Fig. 3. Effect of CCA stem-like cells on MØ-precursors.

A) Migrated CD14+ towards SPH-CM (6h). Relative migrated CD14+cells normalized to migrated CD14+ in presence of MON-CM. Mean $\pm \operatorname{SEM}(n=3, p$ value versus MON) .

B) FACS-profile of CD115, HLA-DR and CD206 expression in MØs obtained by culture of CD14+ with SPH- and MON-CM. Histograms represent three independent experiments with $\mathrm{M} \varnothing$ from three different healthy donors.

C) Relative expression of $\mathrm{M} 1 / \mathrm{M} 2$ and matrix remodeling-related genes. As control $\mathrm{M} \varnothing$ differentiated with M-CSF. GAPDH as internal control. All mRNA levels presented as fold changes normalized to $1(\mathrm{n}=3)$.

D) Adhesion assay using FN-supports. Cells counted and normalized to MON MØs ( $n=3)$. E) SPH M $\varnothing$ invasion assay using Matrigel-coated transwells. Cells counted and normalized to 
migrated MON M $\varnothing s(n=5)$. Mean \pm SEM ( $p$ value versus MON M $\varnothing$ by Student test, * $p \leq 0.05$, $\left.{ }^{* *} p \leq 0.01,{ }^{* * *} p \leq 0.001\right)$.

Fig. 4. Evaluation of TAM-infiltration in mouse model.

A) FACS-profile of mouse CD45-, human CD45+ CD68+cells in isolated mononucleate subset from CCA4-SPH-T and CCA4-MON-T. Upper, Representative dot plots shown. Lower, Table with percent of human CD45 CD68 double positive cells determined by FACS. Data are mean \pm SEM ( $n=3, p$ value versus MON-injected mice by Student $t$ test).

B) Relative gene expression of humanCD14+ isolated from CCA4-T. As control, human CD14+. Human GAPDH as internal control. All mRNA levels presented as fold changes normalized to 1 (mean expression of CCA4 MON-T CD14+ cells). Mean \pm SEM $(n=3, p$ value versus MON-T CD14+ cells). Student t test, ${ }^{*} p \leq 0.05,{ }^{* *} p \leq 0.01,{ }^{* * *} p \leq 0.001$.

Fig. 5. Evaluation of TAM-infiltration in human CCA samples.

A) Quantification of CD163+ cells in both CCA intratumoral (T) and peritumoral (PT) regions. Mean \pm SEM ( $n=23, p$ value versus PT). Distribution of CD163+ cells in CCA lesion ( $L$ ) and tumor front $(F)$ shown by a representative image and corresponding quantification. Mean \pm SEM ( $n=23, p$ value versus $F)$.

B) Correlation of \%CD163+ cells tumor grade (G) $(n=25$, TableS2, One sided Student t-test applied to log ratios in order to compare G1 to G2-G3), as well C) CA19.9 serum levels in CCA patients $(n=17$, TableS2, Pearson correlation between two parameters was calculated using $\mathrm{R}$ and the cortest function, yelding correlation coefficients and $p$ value).

D) Expression of CD115, HLA-DR and CD206 in CCA-infiltrated MØs by FACS included both CCA T and PT regions. Representative histograms of CCA\#23 patient.

E) Relative expression of $\mathrm{M} 1 / \mathrm{M} 2$ and matrix remodeling-related genes. GAPDH as internal control. All mRNA levels displayed as fold changes normalized to 1 (mean expression of PTMØs). Histograms represent the average of three different CCA patients (\#24, \#25, \#26 patients). Mean \pm SEM ( $n=3$, $p$ value versus $P T)$.

F) Gene expression evaluated in a set of CCA patients $(n=23)$ where paired intratumoral epithelial (EPI) and stromal compartments (S) obtained by laser micro-dissection.

Student t test, ${ }^{*} p \leq 0.05,{ }^{* *} p \leq 0.01,{ }^{* * *} p \leq 0.001$.

G) Representative images for IHC analysis of CD163 and CSC-related markers (CD44, EPCAM) in CCA sections (20X) (CCA lesion (L) and tumor front (F)).

Fig. 6. SPH-specific production of bioactive molecules.

A) Representative images show immunohistochemistry of aSMA, CD31, F4/80 and Sirius Red staining for collagen on SPH and MON tumor sections. Scale bar: $200 \mu \mathrm{m}$.

B) Effect of SPH- and MON-CM on CD4 + cells proliferation. CD4+ without stimulation used as control. Data presented as \% of proliferating CD4+ cells. Mean \pm SEM $(n=3, p$ value versus MON-CM by Student $t$ test, $\left.{ }^{*} p \leq 0.05,{ }^{* *} p \leq 0.01,{ }^{* * *} p \leq 0.001\right)$. 
C) Effect of SPH- and MON-CM on HUVEC tube formation. HUCCT1 and SG231 MON- and SPH-CM were added to the medium of HUVEC to assay their effect on the tube formation ability of HUVEC. Data presented as Number of branches/well. Mean $\pm \operatorname{SEM}(n=3, p$ value versus MON-CM by Student t test, $\left.{ }^{*} p \leq 0.05,{ }^{* *} p \leq 0.01,{ }^{* * *} p \leq 0.001\right)$.

D) Heat map representation of soluble mediators released by SPH and MON (ELISA). Concentration as $\mathrm{pg} / \mathrm{mL}$. Molecules clustered with names shown on right of heat map. Each raw corresponds to a single compound, and each column represents an independent condition. Heat-map color scale corresponds to relative molecule expression (on the top, minimum and maximum of all values). Results are average of three independent experiments. E) Relative expression of transcript-encoding receptors for IL4 (IL4-R), IL13 (IL13Ra1, IL13Ra2), OA (CD44, ITGA5, ITGB3, SDC4) and IL34 (SDC1) in SPH and MON MØ. CD14+ cells as well MCSF derived MØ, as controls. GAPDH as internal control. All mRNA levels are presented as fold changes normalized to 1 (mean expression of MON MØ).

F) IL13, OA and IL34 levels in CCA patients $(n=12)$ and controls (CTR) $(n=12)$ serum levels. Data are mean \pm SEM ( $p$ value versus MON M $\varnothing$ by Student $t$ test, ${ }^{*} p \leq 0.05,{ }^{* *} p \leq 0.01,{ }^{* * *}$ $\mathrm{p} \leq 0.001)$.

Fig. 7. IL13, OA, IL34 combination mimics SPH-like effects on MØ-differentiation and monocyte recruitment.

A) FACS-profile of CD115, HLA-DR and CD206 expression in MØs obtained by CD14+ cultured in presence of IL13 $(80 \mathrm{pg} / \mathrm{mL}), O A(0,9 \mathrm{ng} / \mathrm{mL})$ and IL34 $(80 \mathrm{pg} / \mathrm{mL})$, added to the MON-CM (in green). Inhibitory impact of human antibodies anti IL13 (800ng/mL, 10.000X), OA $(2700 \mathrm{ng} / \mathrm{mL}, 3000 \mathrm{X})$, IL34 (800ng/mL, 10.000X) alone (in brown, dark grey and dark green, respectively) or in combination was shown (in violet). Effects of both SPH- (in red) and MON- (in blue) CM also shown. Histograms represent three independent experiments using $\mathrm{M} \varnothing$ from three different healthy donors.

B) Relative expression of M1/M2 and matrix remodeling-related genes. GAPDH as internal control. All mRNA levels displayed as fold changes normalized to 1 (mean expression of MON MØ) $(n=3)$. Mean \pm SEM ( $p$ value versus MON- or SPH-CM by Student test, * $p \leq 0.05$, $\left.{ }^{* *} p \leq 0.01,{ }^{* * *} p \leq 0.001\right)$.

C) Invasion and adhesion assay with FN-supports. Cells counted normalized to MON $\operatorname{MØs}(n=5)$. Migration assay of monocytes. Monocytes counted and normalized to monocyte migrated in presence of MON-CM $(n=5)$. Mean \pm SEM ( $p$ value versus MON- or SPH-CM by Student t test, $\left.{ }^{*} p \leq 0.05,{ }^{* *} p \leq 0.01,{ }^{* * *} p \leq 0.001\right)$.

D) MØ-role in supporting in vivo tumorigenicity. 1,000 MONs (SG231) co-injected with 300 in vitro-educated MØs into NSG mice. Tumor growth evaluated. Mean $\pm S E M(n=5, p$ value versus MON or SPH M $\varnothing$ at week 13 by Student $t$ test, $\left.{ }^{*} p \leq 0.05,{ }^{* \star} p \leq 0.01,{ }^{* \star} p \leq 0.001\right)$. 


\section{EXPERIMENTAL PROCEDURES}

\section{Mice}

The Humanitas Animal Care and Research Advisory Committee approved housing and experimental animal procedures. NOD/SCID/IL2ry null (NSG) mice (Jackson Laboratory) were_used in all experiments. The mice were used at 6 weeks of age, in accordance with the guidelines and with the approval of the local Experimental Animal Committee [16].

\section{Sphere Assay}

The cells were grown in anchoring-independent conditions with selective serum-free DMEM/F12 medium supplemented with 1X B27 supplement minus vitamin A (Life Technologies), human recombinant EGF (hrEGF) (R\&D System) $(20 \mathrm{ng} / \mathrm{mL})$, and bFGF (R\&D System) (20 ng/mL) for 15 days.

\section{Monocyte Isolation and Macrophage Differentiation}

Human monocytes were obtained from healthy blood donor buffy coats by gradient centrifugation using a Ficoll gradient (GE Healthcare) and further purified from peripheral blood mononuclear cells (PBMC) by magnetic-activated cell sorting (MACS) using CD14 microbeads (Miltenyi Biotech). After MACS purification, two fractions were obtained: the CD14+ fraction and the eluate (composed of all PBMC CD14- cells). The purity of CD14+ cells was $>90 \%$. CD14+ cells were cultured in presence of $30 \%$ CCA sphere- or monolayer conditioned medium (CM).

\section{In Vivo Study}

In vivo experiments were performed in accordance with the guidelines and approval of the local Experimental Animal Committee. The following materials were used: 1,000 sphere- or monolayer-derived cells to measure the tumorigenic potential of CCA cells and 1,000 monolayer-derived cells and 300 macrophages for TAMs + CCA monolayer co-injection experiments. The cells were dissociated into single-cell suspensions and resuspended in 100 $\mu \mathrm{L}$ of DMEM and reduced Matrigel growth factor (BD Bioscience) (1:1), and the mixture was s.c. injected into the right flank of 6-week-old NSG mice. Tumor growth was monitored, and the diameter of the growing tumors was measured in millimeters every week using a caliper. The animals were sacrificed when the xenografts reached $2.0 \mathrm{~cm}$ in diameter $[15,16,26]$. The limiting dilution analysis was performed by sorting 1,000/100/10 alive cells from dissociated CCA monolayer and spheres. Web-based Extreme Limiting Dilution Analysis (ELDA) statistical software (http://bioinf.wehi.edu.au/software/limdil/index.html) was used [28].

\section{Patient Samples}

All patients gave written, informed consent. Study was approved by local institutional review boards. CCA samples and peritumoral non-cancerous liver were obtained from patients submitted to surgical resections (Department of Hepatobiliary Surgery, Istituto Clinico 
Humanitas). Distinctions between intrahepatic CCA and perihilar CCA were based on clinical records including surgical reports.

Additional procedures in "Supplementary Information".

\section{FOOTNOTE PAGE}

Acknowledgements We thank Dr. Demetris .A.J. (University of Pittsburgh, Pittsburgh, PA) for HUCCT1, CCLP1 and SG231 cells; Dr. Gores G. J. (Mayo Clinic, Rochester, MN) for human immortalized non-malignant cholangiocyte cell line H69; Dr. Morenghi E. for assistance with the statistical analysis; Dr. Trombetta E. for human anti CD44 and EPCAM antibodies. This material is the result of work supported by resources at the Central Texas Veterans Health Care System. The views expressed in this article are those of the authors and do not necessarily represent the views of the Department of Veterans Affairs.

\section{References}

[1] Andersen JB, Spee B, Blechacz BR, Avital I, Komuta M, Barbour A, et al. Genomic and genetic characterization of cholangiocarcinoma identifies therapeutic targets for tyrosine kinase inhibitors. Gastroenterology 2012;142:1021-1031 e1015.

[2] Blechacz B, Gores GJ. Cholangiocarcinoma: advances in pathogenesis, diagnosis, and treatment. Hepatology 2008;48:308-321.

[3] Raggi C, Invernizzi P, Andersen JB. Impact of microenvironment and stem-like plasticity in cholangiocarcinoma: molecular networks and biological concepts. J Hepatol 2015;62:198-207.

[4] Banales JM, Cardinale V, Carpino G, Marzioni M, Andersen JB, Invernizzi P, et al. Expert consensus document: Cholangiocarcinoma: current knowledge and future perspectives consensus statement from the European Network for the Study of Cholangiocarcinoma (ENS-CCA). Nat Rev Gastroenterol Hepatol 2016;13:261-280.

[5] Lin G, Liu Y, Li S, Mao Y, Wang J, Shuang Z, et al. Elevated neutrophil-to-lymphocyte ratio is an independent poor prognostic factor in patients with intrahepatic cholangiocarcinoma. Oncotarget 2016.

[6] Sabbatino F, Villani V, Yearley JH, Deshpande V, Cai L, Konstantinidis IT, et al. PD-L1 and HLA Class I Antigen Expression and Clinical Course of the Disease in Intrahepatic Cholangiocarcinoma. Clin Cancer Res 2016;22:470-478.

[7] Mao ZY, Zhu GQ, Xiong M, Ren L, Bai L. Prognostic value of neutrophil distribution in cholangiocarcinoma. World J Gastroenterol 2015;21:4961-4968.

[8] Sasaki M, Tsuneyama K, Ishikawa A, Nakanuma Y. Intrahepatic cholangiocarcinoma in cirrhosis presents granulocyte and granulocyte-macrophage colony-stimulating factor. Hum Pathol 2003;34:1337-1344. 
[9] Fan B, Malato Y, Calvisi DF, Naqvi S, Razumilava N, Ribback S, et al. Cholangiocarcinomas can originate from hepatocytes in mice. J Clin Invest 2012;122:2911-2915.

[10] Marquardt JU, Raggi C, Andersen JB, Seo D, Avital I, Geller D, et al. Human hepatic cancer stem cells are characterized by common stemness traits and diverse oncogenic pathways. Hepatology 2011;54:1031-1042.

[11] Raggi C, Factor VM, Seo D, Holczbauer A, Gillen MC, Marquardt JU, et al. Epigenetic reprogramming modulates malignant properties of human liver cancer. Hepatology 2014;59:2251-2262.

[12] Kreso A, Dick JE. Evolution of the cancer stem cell model. Cell Stem Cell 2014;14:275291.

[13] Hanahan D, Coussens LM. Accessories to the crime: functions of cells recruited to the tumor microenvironment. Cancer Cell 2012;21:309-322.

[14] Plaks V, Kong N, Werb Z. The cancer stem cell niche: how essential is the niche in regulating stemness of tumor cells? Cell Stem Cell 2015;16:225-238.

[15] Jinushi M, Chiba S, Yoshiyama H, Masutomi K, Kinoshita I, Dosaka-Akita H, et al. Tumor-associated macrophages regulate tumorigenicity and anticancer drug responses of cancer stem/initiating cells. Proc Natl Acad Sci U S A 2011;108:12425-12430.

[16] Su S, Liu Q, Chen J, Chen F, He C, Huang D, et al. A positive feedback loop between mesenchymal-like cancer cells and macrophages is essential to breast cancer metastasis. Cancer Cell 2014;25:605-620.

[17] Wan S, Zhao E, Kryczek I, Vatan L, Sadovskaya A, Ludema G, et al. Tumor-associated macrophages produce interleukin 6 and signal via STAT3 to promote expansion of human hepatocellular carcinoma stem cells. Gastroenterology 2014;147:1393-1404.

[18] Lu H, Clauser KR, Tam WL, Frose J, Ye X, Eaton EN, et al. A breast cancer stem cell niche supported by juxtacrine signalling from monocytes and macrophages. Nat Cell Biol 2014;16:1105-1117.

[19] Zhou W, Ke SQ, Huang Z, Flavahan W, Fang X, Paul J, et al. Periostin secreted by glioblastoma stem cells recruits M2 tumour-associated macrophages and promotes malignant growth. Nat Cell Biol 2015;17:170-182.

[20] Biswas SK, Sica A, Lewis CE. Plasticity of macrophage function during tumor progression: regulation by distinct molecular mechanisms. J Immunol 2008;180:20112017.

[21] Roberts PE. Isolation and establishment of human tumor stem cells. Methods Cell Biol 2008;86:325-342.

[22] Pollard SM, Yoshikawa K, Clarke ID, Danovi D, Stricker S, Russell R, et al. Glioma stem cell lines expanded in adherent culture have tumor-specific phenotypes and are suitable for chemical and genetic screens. Cell Stem Cell 2009;4:568-580.

[23] Miyagiwa M, Ichida T, Tokiwa T, Sato J, Sasaki H. A new human cholangiocellular carcinoma cell line (HuCC-T1) producing carbohydrate antigen 19/9 in serum-free medium. In Vitro Cell Dev Biol 1989;25:503-510.

[24] Han C, Wu T. Cyclooxygenase-2-derived prostaglandin E2 promotes human cholangiocarcinoma cell growth and invasion through EP1 receptor-mediated activation of the epidermal growth factor receptor and Akt. J Biol Chem 2005;280:24053-24063.

[25] Shimizu Y, Demetris AJ, Gollin SM, Storto PD, Bedford HM, Altarac S, et al. Two new human cholangiocarcinoma cell lines and their cytogenetics and responses to growth 
factors, hormones, cytokines or immunologic effector cells. Int J Cancer 1992;52:252260.

[26] Cardinale V, Renzi A, Carpino G, Torrice A, Bragazzi MC, Giuliante F, et al. Profiles of cancer stem cell subpopulations in cholangiocarcinomas. Am J Pathol 2015;185:17241739.

[27] Sirica AE, Zhang Z, Lai GH, Asano T, Shen XN, Ward DJ, et al. A novel "patient-like" model of cholangiocarcinoma progression based on bile duct inoculation of tumorigenic rat cholangiocyte cell lines. Hepatology 2008;47:1178-1190.

[28] Saeed AI, Sharov V, White J, Li J, Liang W, Bhagabati N, et al. TM4: a free, opensource system for microarray data management and analysis. Biotechniques 2003;34:374-378.

[29] Quintana E, Shackleton M, Sabel MS, Fullen DR, Johnson TM, Morrison SJ. Efficient tumour formation by single human melanoma cells. Nature 2008;456:593-598.

[30] Raggi C, Mousa HS, Correnti M, Sica A, Invernizzi P. Cancer stem cells and tumorassociated macrophages: a roadmap for multitargeting strategies. Oncogene 2015.

[31] Sica A, Schioppa T, Mantovani A, Allavena P. Tumour-associated macrophages are a distinct M2 polarised population promoting tumour progression: potential targets of anticancer therapy. Eur J Cancer 2006;42:717-727.

[32] Steidl C, Lee T, Shah SP, Farinha P, Han G, Nayar T, et al. Tumor-associated macrophages and survival in classic Hodgkin's lymphoma. N Engl J Med 2010;362:875885.

[33] Bingle L, Brown NJ, Lewis CE. The role of tumour-associated macrophages in tumour progression: implications for new anticancer therapies. J Pathol 2002;196:254-265.

[34] Schmidt T, Brodesser A, Schnitzler N, Gruger T, Brandenburg K, Zinserling J, et al. CD66b Overexpression and Loss of C5a Receptors as Surface Markers for Staphylococcus aureus-Induced Neutrophil Dysfunction. PLoS One 2015;10:e0132703.

[35] Solinas G, Schiarea S, Liguori M, Fabbri M, Pesce S, Zammataro L, et al. Tumorconditioned macrophages secrete migration-stimulating factor: a new marker for $\mathrm{M} 2$ polarization, influencing tumor cell motility. J Immunol 2010;185:642-652.

[36] Chen J, Yao Y, Gong C, Yu F, Su S, Liu B, et al. CCL18 from tumor-associated macrophages promotes breast cancer metastasis via PITPNM3. Cancer Cell 2011;19:541-555.

[37] Pollard JW. Trophic macrophages in development and disease. Nat Rev Immunol 2009;9:259-270.

[38] Biswas SK, Mantovani A. Macrophage plasticity and interaction with lymphocyte subsets: cancer as a paradigm. Nat Immunol 2010;11:889-896.

[39] Sica A, Mantovani A. Macrophage plasticity and polarization: in vivo veritas. J Clin Invest 2012;122:787-795.

[40] Dang W, Qin Z, Fan S, Wen Q, Lu Y, Wang J, et al. miR-1207-5p suppresses lung cancer growth and metastasis by targeting CSF1. Oncotarget 2016.

[41] Mantovani A, Allavena P. The interaction of anticancer therapies with tumor-associated macrophages. J Exp Med 2015;212:435-445.

[42] Franklin RA, Liao W, Sarkar A, Kim MV, Bivona MR, Liu K, et al. The cellular and molecular origin of tumor-associated macrophages. Science 2014;344:921-925.

[43] Van Overmeire E, Laoui D, Keirsse J, Van Ginderachter JA, Sarukhan A. Mechanisms driving macrophage diversity and specialization in distinct tumor microenvironments and parallelisms with other tissues. Front Immunol 2014;5:127. 
[44] Locatelli L, Cadamuro M, Spirli C, Fiorotto R, Lecchi S, Morell CM, et al. Macrophage recruitment by fibrocystin-defective biliary epithelial cells promotes portal fibrosis in congenital hepatic fibrosis. Hepatology 2016;63:965-982.

[45] Felix J, Elegheert J, Gutsche I, Shkumatov AV, Wen Y, Bracke N, et al. Human IL-34 and CSF-1 establish structurally similar extracellular assemblies with their common hematopoietic receptor. Structure 2013;21:528-539.

[46] Kuan CT, Wakiya K, Dowell JM, Herndon JE, 2nd, Reardon DA, Graner MW, et al. Glycoprotein nonmetastatic melanoma protein B, a potential molecular therapeutic target in patients with glioblastoma multiforme. Clin Cancer Res 2006;12:1970-1982.

[47] Rose AA, Pepin F, Russo C, Abou Khalil JE, Hallett M, Siegel PM. Osteoactivin promotes breast cancer metastasis to bone. Mol Cancer Res 2007;5:1001-1014.

[48] De Minicis S, Kisseleva T, Francis H, Baroni GS, Benedetti A, Brenner D, et al. Liver carcinogenesis: rodent models of hepatocarcinoma and cholangiocarcinoma. Dig Liver Dis 2013;45:450-459.

Author names in bold designate shared co-first authorship 


\section{Table 1}

Correlation of SPH stem-like genes and OA, IL34 and IL13 in clinical CCA ( $n=104$ patient tumors) using a microarray database (1). Pearson correlation between gene pairs was calculated using $R$ and the "cortest" function, yielding correlation coefficients and $p$-values

\begin{tabular}{|c|c|c|}
\hline \multirow[t]{3}{*}{ and OA, IL34 anc } & $\begin{array}{l}\text { stem-Iike } \\
\text { CCA (n= }\end{array}$ & tumors) \\
\hline & \multicolumn{2}{|c|}{$O A$} \\
\hline & $\mathrm{R}$ & $p$ value \\
\hline BMI1 & 0.3504 & 0.0003 \\
\hline CD44 & 0.3844 & 0.0001 \\
\hline CTNNB1 & 0.4419 & 0.0000 \\
\hline KITLG & 0.3975 & 0.0000 \\
\hline KLF4 & 0.3603 & 0.0001 \\
\hline LEF1 & 0.6321 & 0.0000 \\
\hline THY1 & 0.5118 & 0.0000 \\
\hline \multirow{2}{*}{ SPH Genes } & \multicolumn{2}{|c|}{ IL34 } \\
\hline & $\mathrm{R}$ & $p$ value \\
\hline MAML1 & 0.3423 & 0.0004 \\
\hline THY1 & 0.2966 & 0.0022 \\
\hline \multirow{2}{*}{ SPH Genes } & \multicolumn{2}{|c|}{ IL13 } \\
\hline & $\mathrm{R}$ & $p$ value \\
\hline LIN28A & 0.4906 & 0.0000 \\
\hline POU5F1 & 0.2685 & 0.0059 \\
\hline sox2 & 0.3121 & 0.0013 \\
\hline
\end{tabular}


Fig.1

A

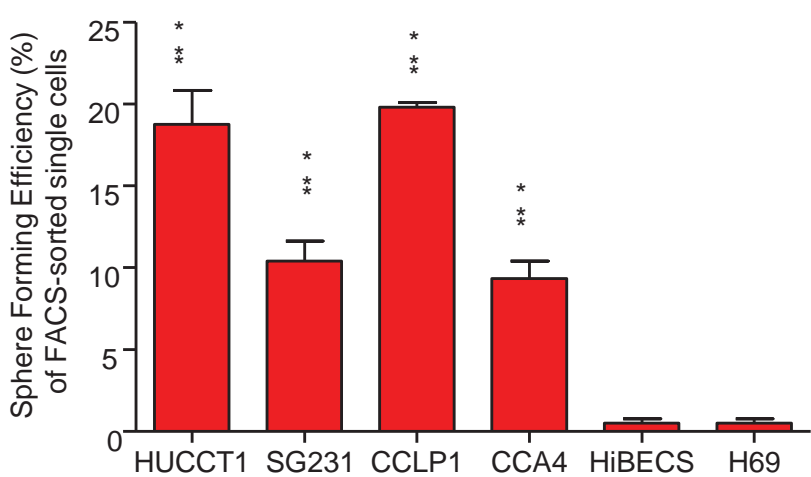

C

\section{Generated Tumors (\%)}

\begin{tabular}{cccc} 
Cell Line & MON & SPH & $\boldsymbol{p}$ value \\
\hline HUCCT1 & $60(3 / 5)$ & $100(5 / 5)$ & 0.0003 \\
\hline SG231 & $40(2 / 5)$ & $100(5 / 5)$ & 0.0000 \\
\hline CCLP1 & $60(3 / 5)$ & $100(4 / 4)$ & 0.0003 \\
CCA4 & $50(2 / 4)$ & $100(4 / 4)$ & 0.0000 \\
\hline \multicolumn{4}{c}{ The data are mean \pm SEM }
\end{tabular}

( $p$ value versus MON by Student $t$ test)
B
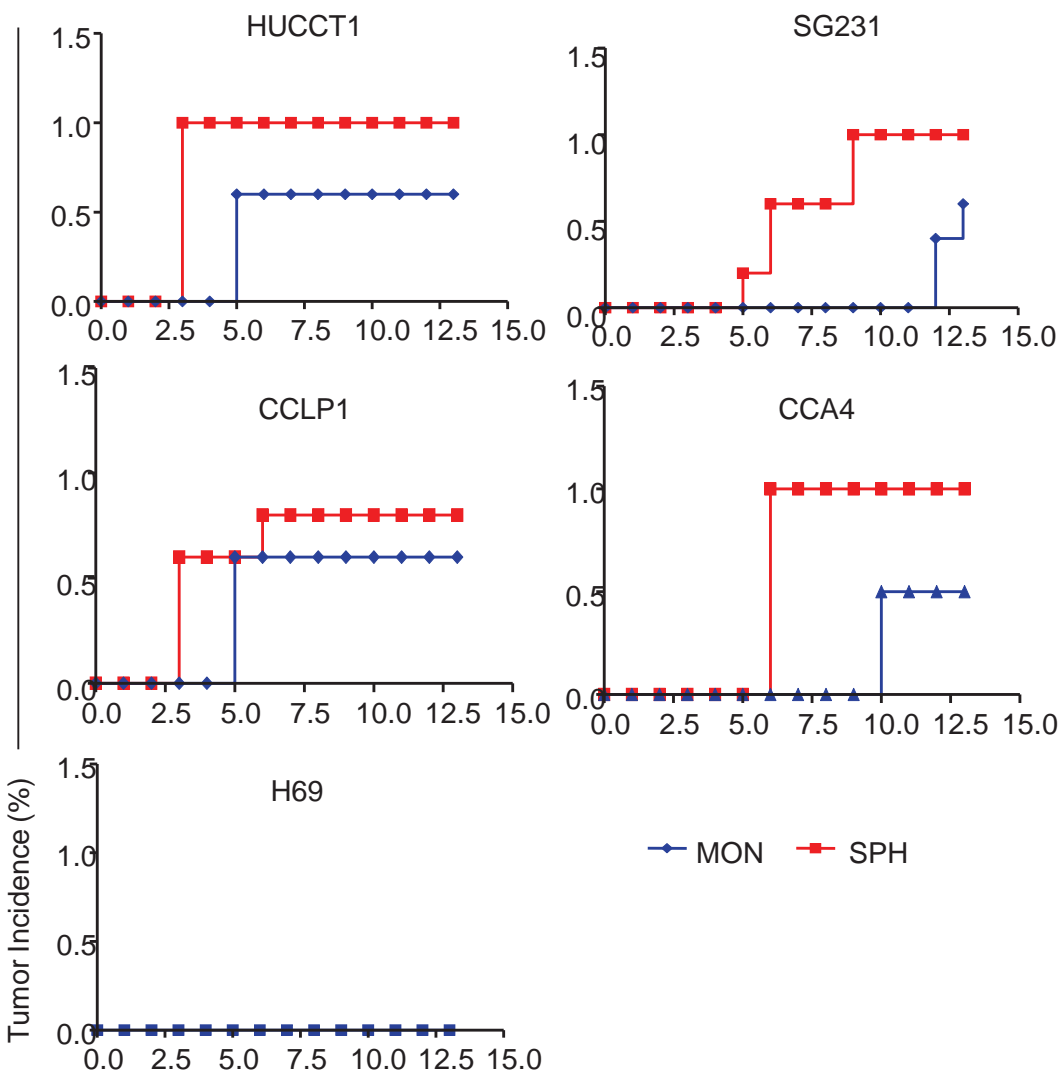

Weeks

D
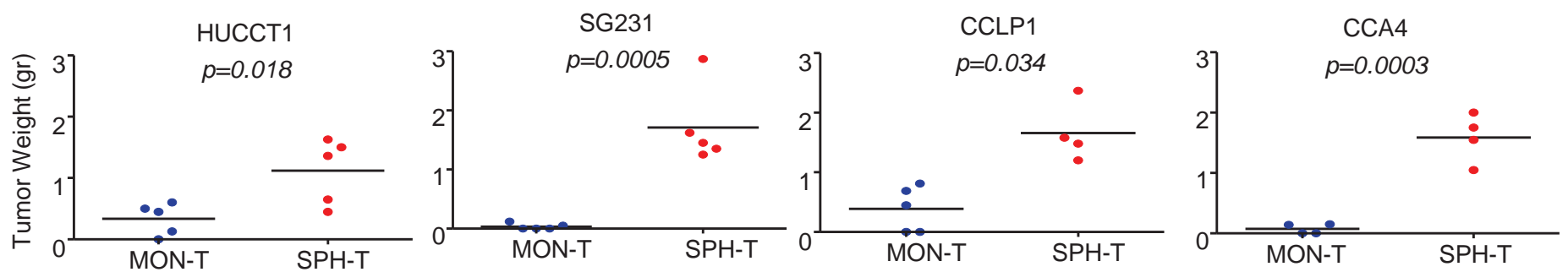

E

\begin{tabular}{|c|c|c|c|c|c|}
\hline $\mathrm{N}^{\circ}$ of injected cells & 1000 & 100 & 10 & \multirow{2}{*}{$\begin{array}{c}\text { Tumor Initiating Fraction (TIF) } \\
(95 \% \mathrm{Cl})\end{array}$} & \multirow{2}{*}{$p$ value } \\
\hline & \multicolumn{3}{|c|}{$\begin{array}{l}\mathrm{N}^{\circ} \text { of tumors formed/ } \\
\text { total } \mathrm{n}^{\circ} \text { of transplants }\end{array}$} & & \\
\hline CCLP1 MON & $3 / 5$ & $3 / 6$ & $1 / 6$ & $1 / 505(1 / 1295-1 / 197)$ & \\
\hline CCLP1 SPH & $4 / 4$ & $5 / 6$ & $5 / 6$ & $1 / 25(1 / 69-1 / 9)$ & \\
\hline
\end{tabular}
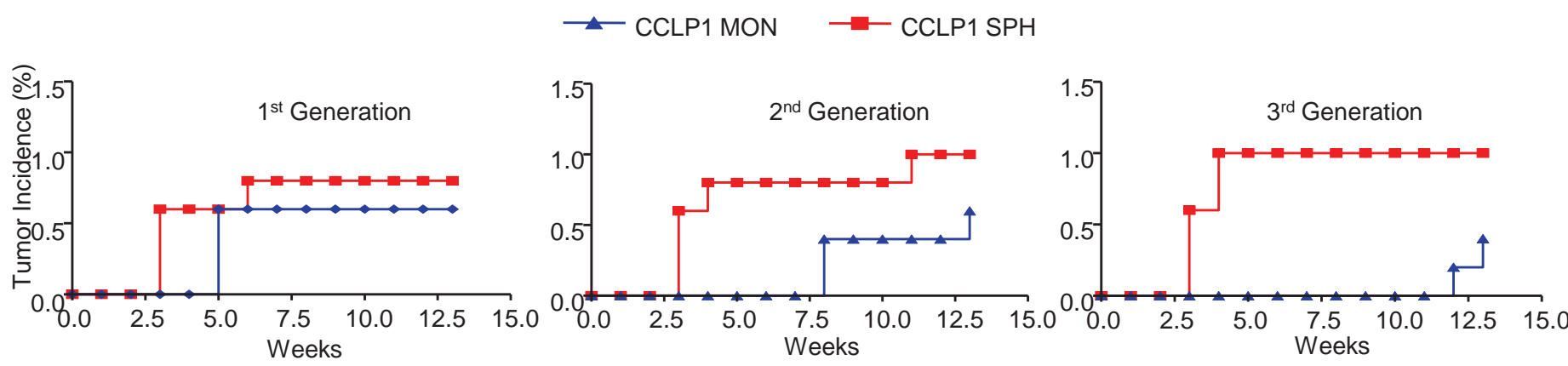
Fig.2

A

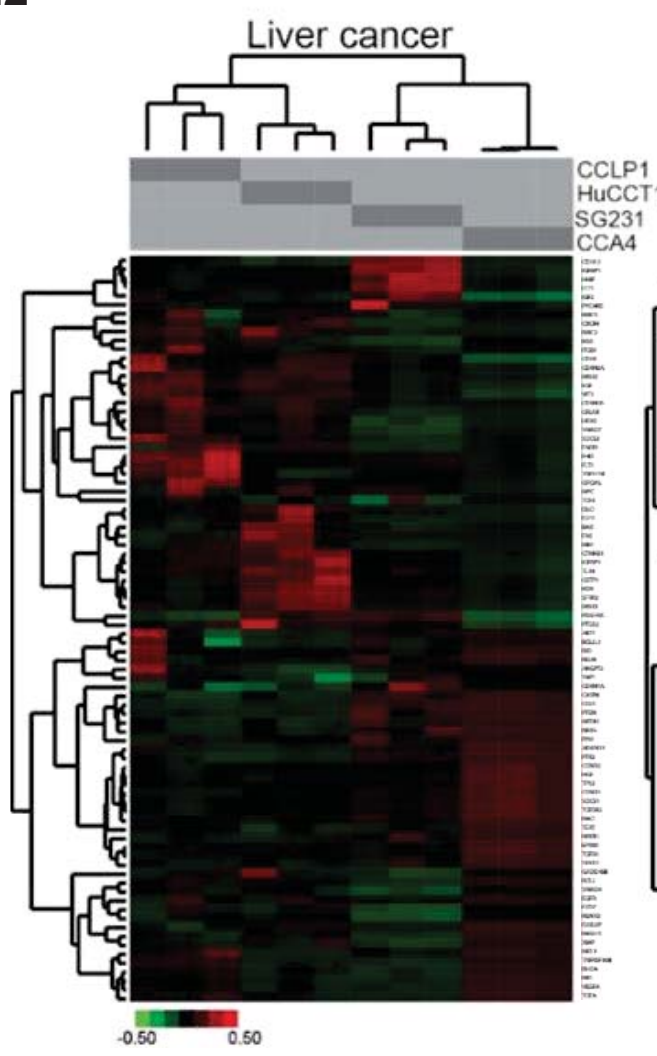

CSC

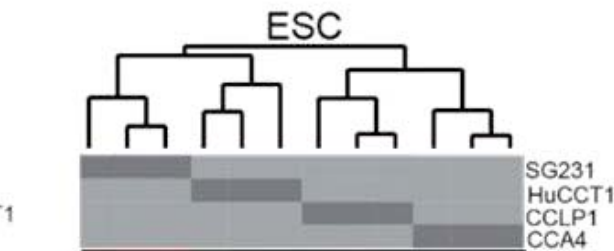

B

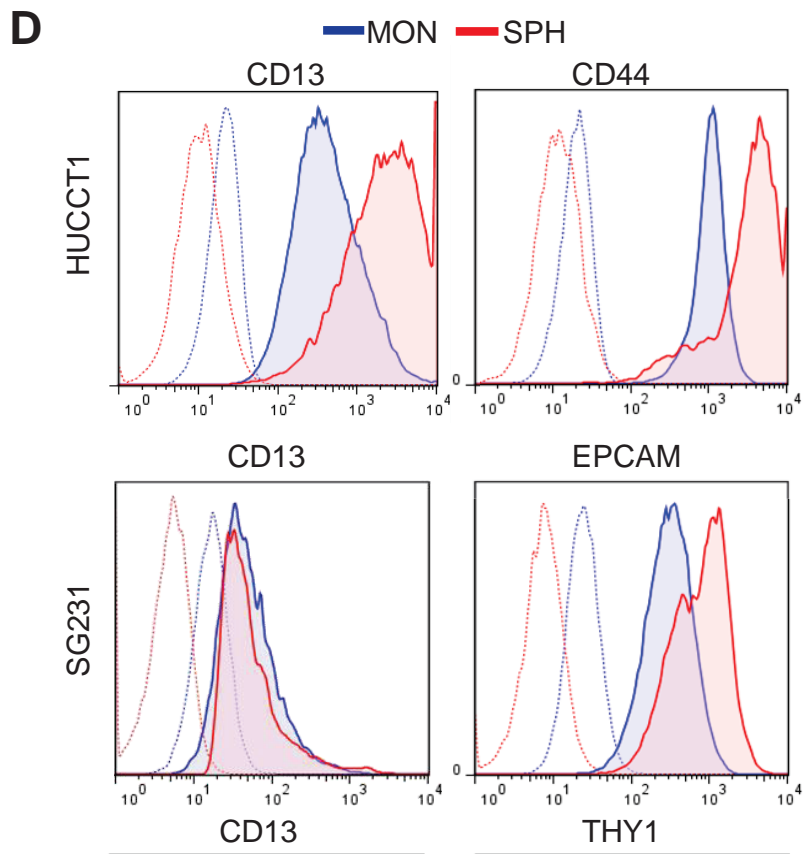

BCL2L1,

CDKN1A,

CTNNB1,

IGF2,

ITGB1,

LEF1

\section{Common CCA-SPH Deregulated Genes}

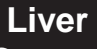

Cancer

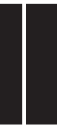

G2, BMI1, BMP7,

CD24, CD44, EPCAM,

FGFR2, KITLG, KLF4, LATS1, LIN28A,

MAML1, MYC, NFKB1,

NOS2, NOTCH1,

POU5F1, PROM1,

SOX2, STAT3,

TGFBR1, THY1,YAP1

C

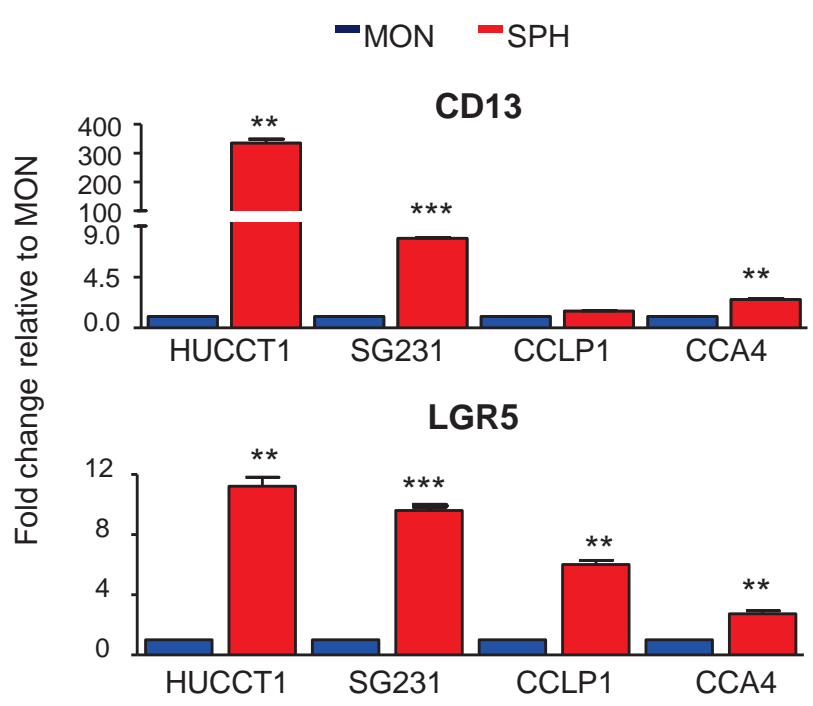

ESC

HNF4A
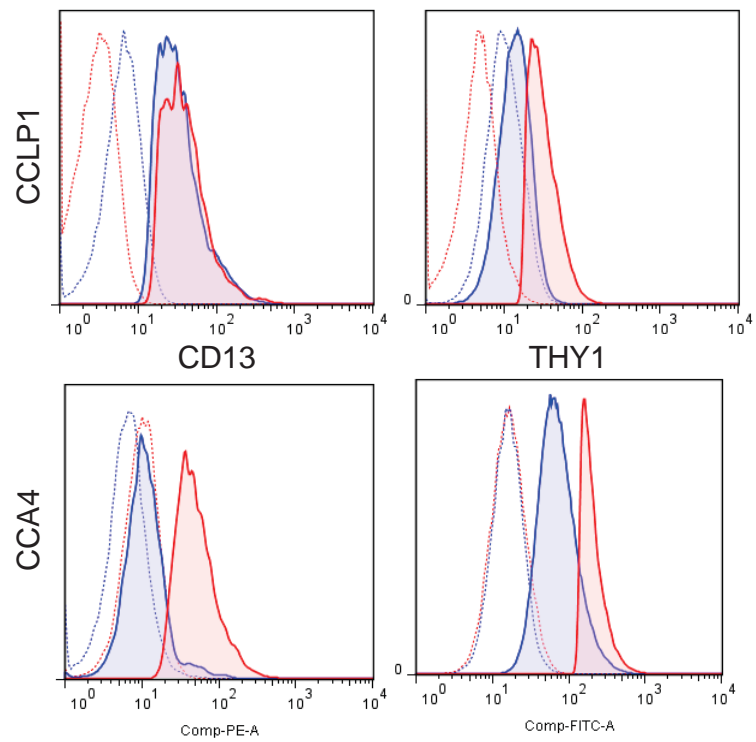
Fig.3

A

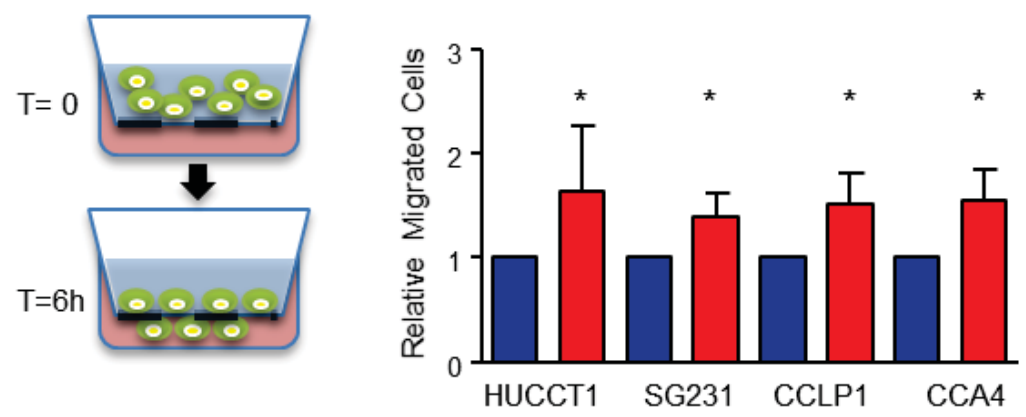

B
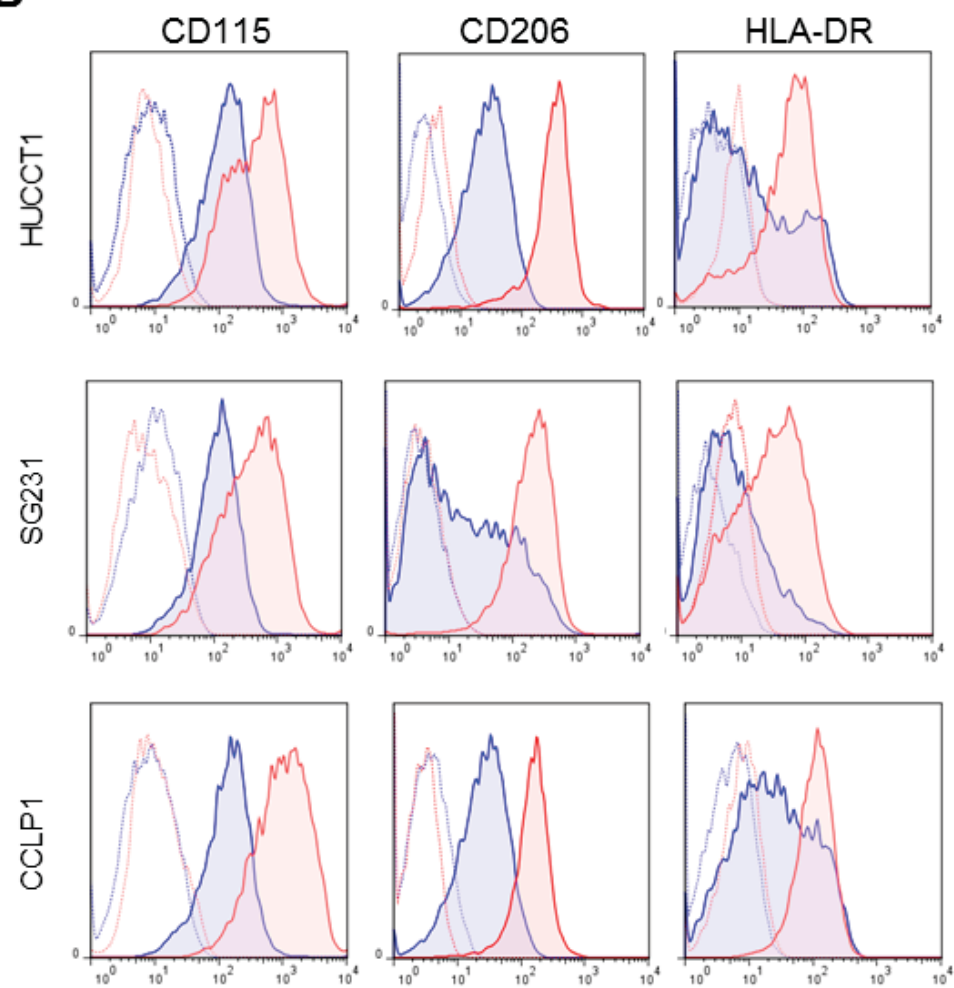

4
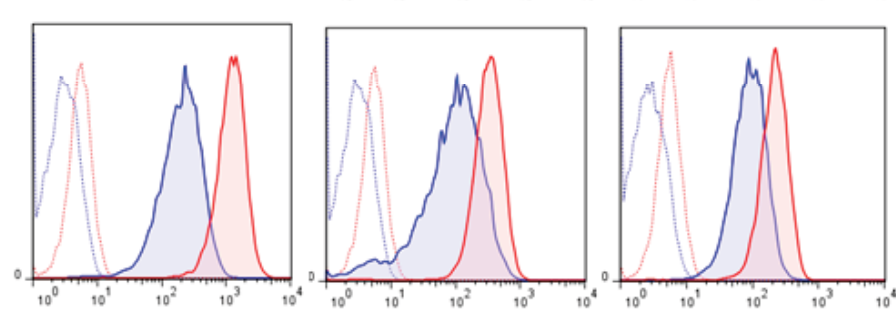

MON-CM $\simeq$ SPH-CM

D

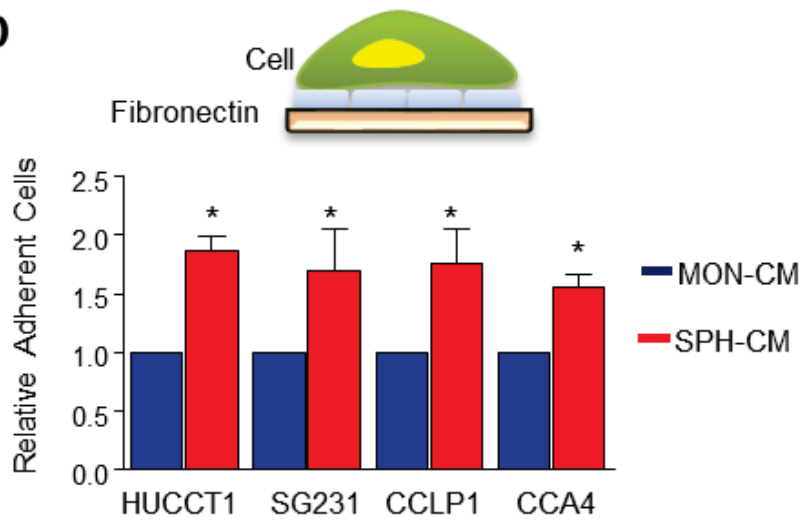

C $-\mathrm{MON}-\mathrm{CM}=\mathrm{SPH}-\mathrm{CM}=\mathrm{M}-\mathrm{CSF}$
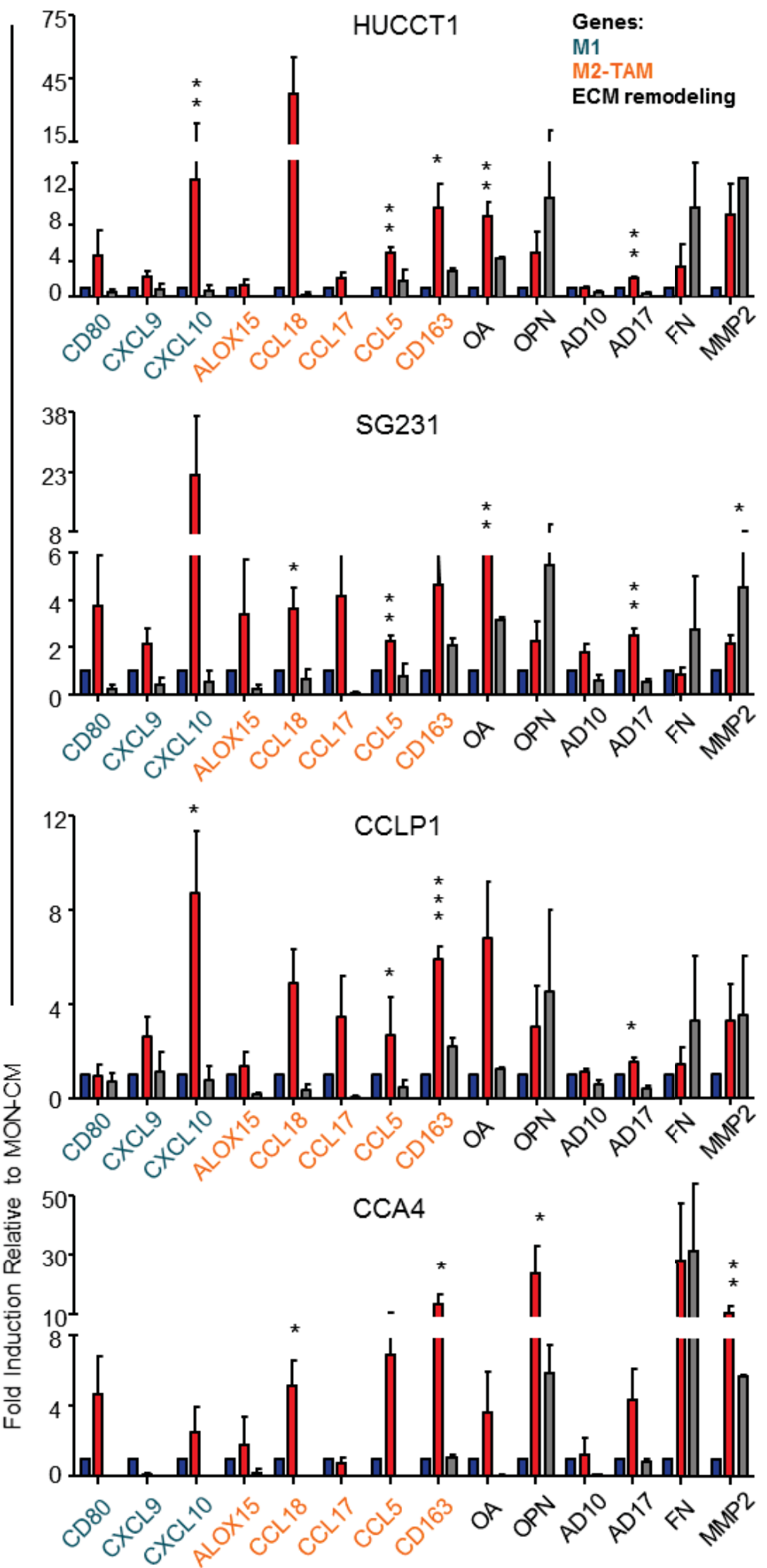

E

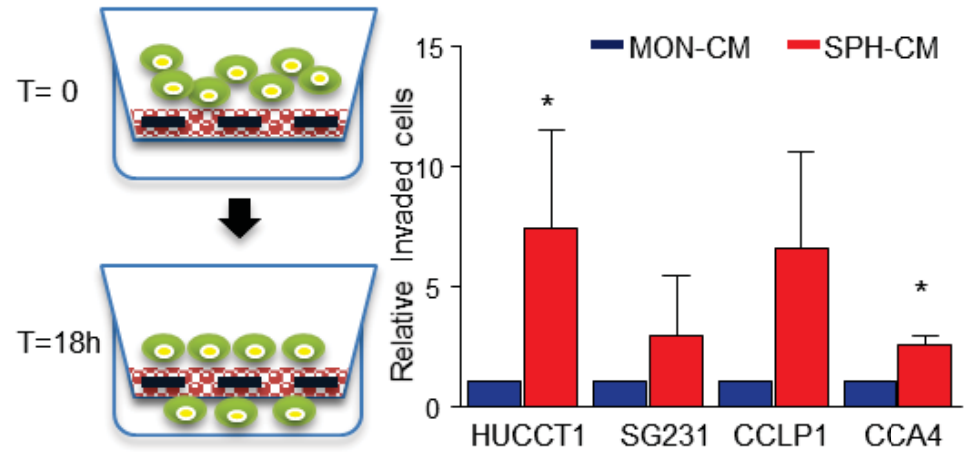


Fig.4

A Mouse CCA4 SPH-T
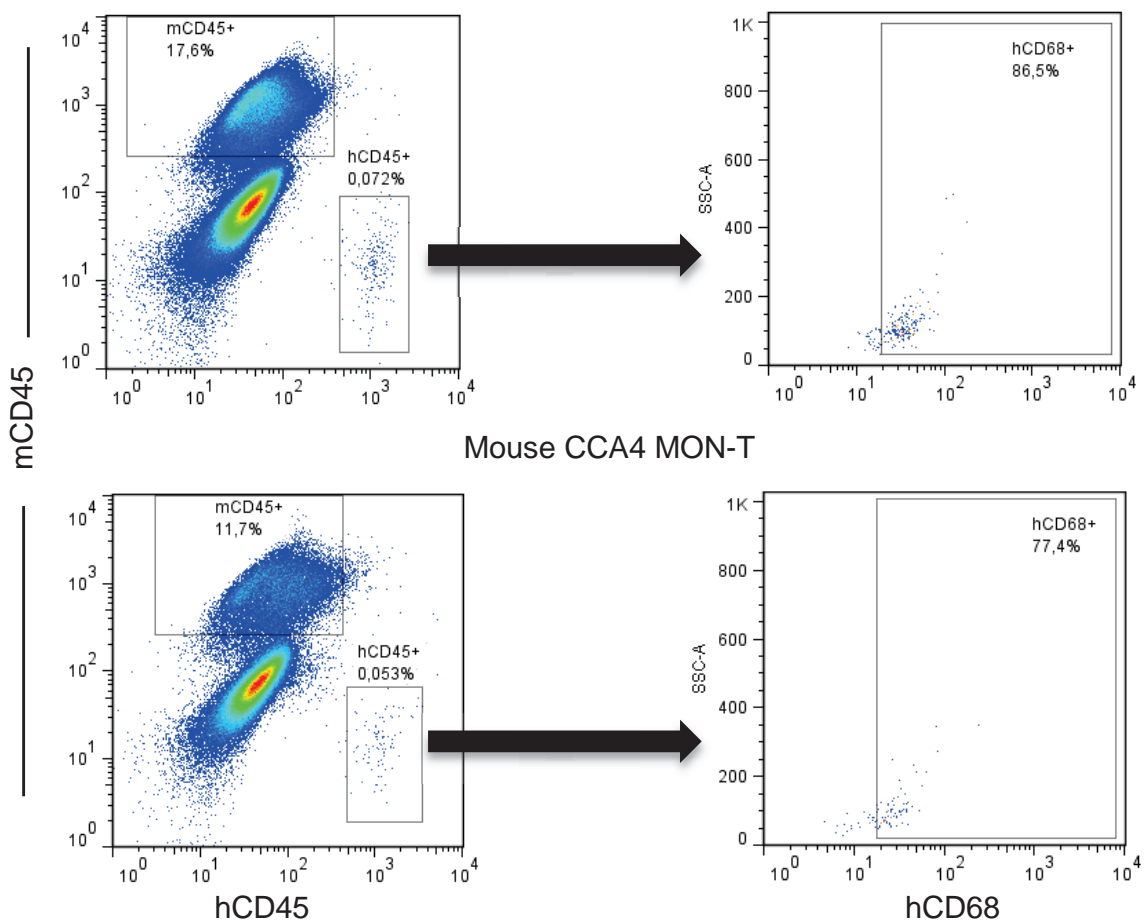

$\begin{array}{ccccc}\begin{array}{c}\text { S.c. } \\ \text { injected cells }\end{array} & \begin{array}{c}\text { \% hCD45+ } \\ (\text { Mean } \pm \text { SEM) }\end{array} & p \text { value } & \begin{array}{c}\text { \% hCD45+ hCD68+ } \\ \text { (Mean } \pm \text { SEM) }\end{array} & p \text { value } \\ \text { CCA4 SPH } & 0.068 \pm 0.006 & & 83.55 \pm 2.19 & \\ & & 0.0405 & & 0.0072 \\ \text { CCA4 MON } & 0.048 \pm 0.008 & & 70.00 \pm 2.83 & \end{array}$

The data are mean \pm SEM ( $n=3, p$ value versus MON by Student $t$ test)

B

MON-T hCD14 SPH-T hCD14 hCD14

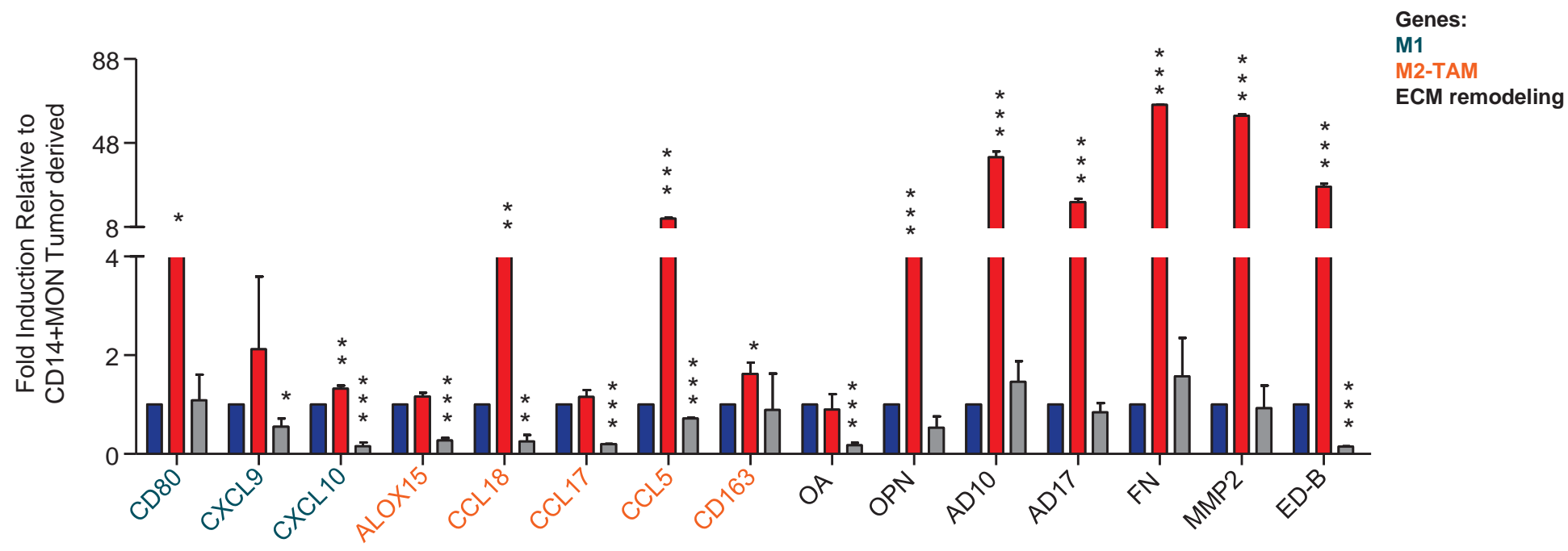


Fig.5

A

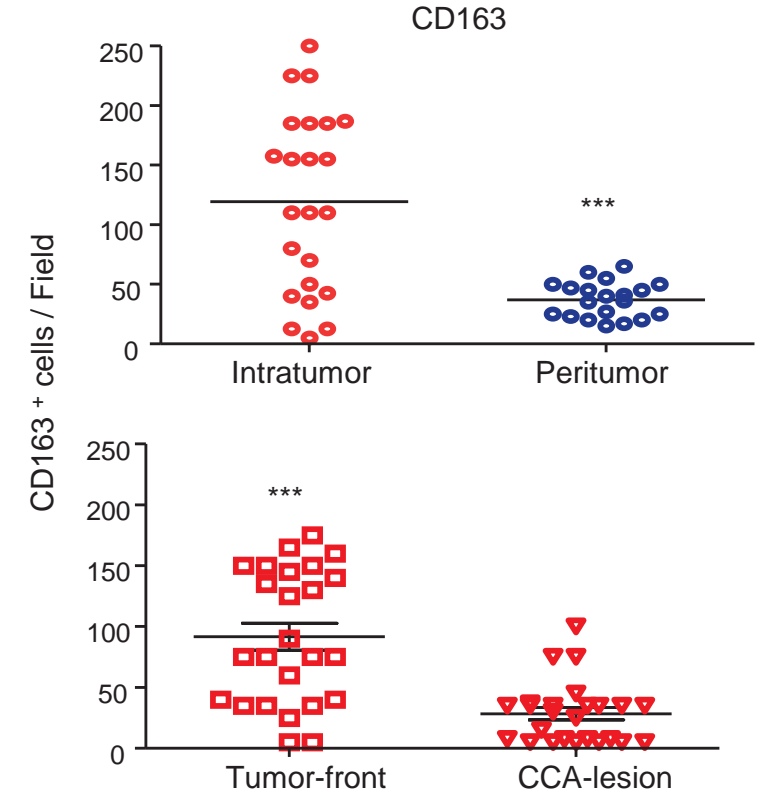

B

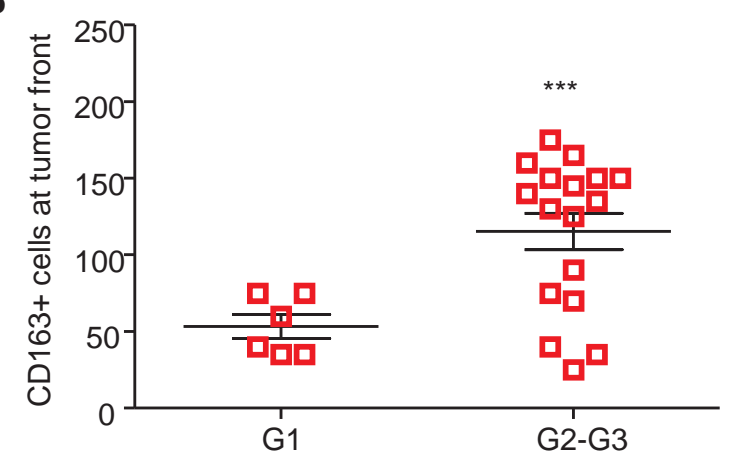

C

Pearson $r=0.636$

$p$ value $=0.0061$

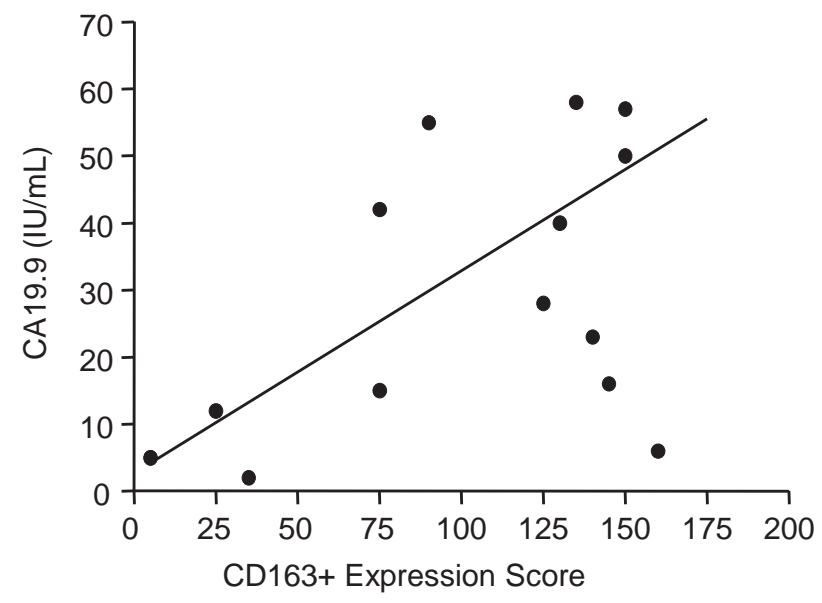

D CCA\#23 T CCA\#23 PT
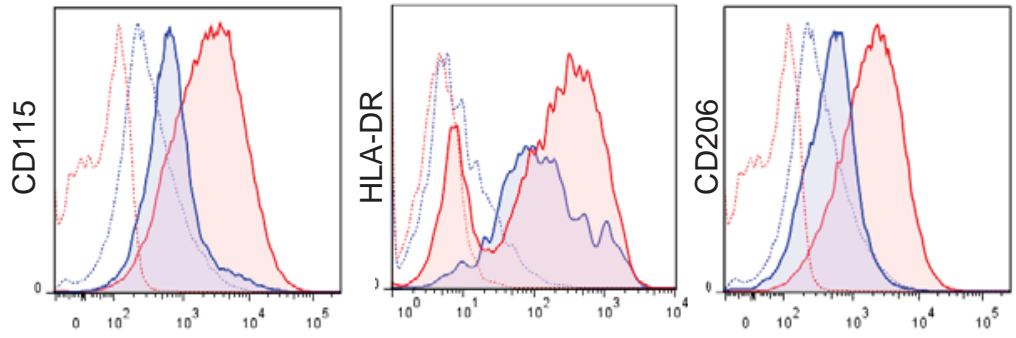

E

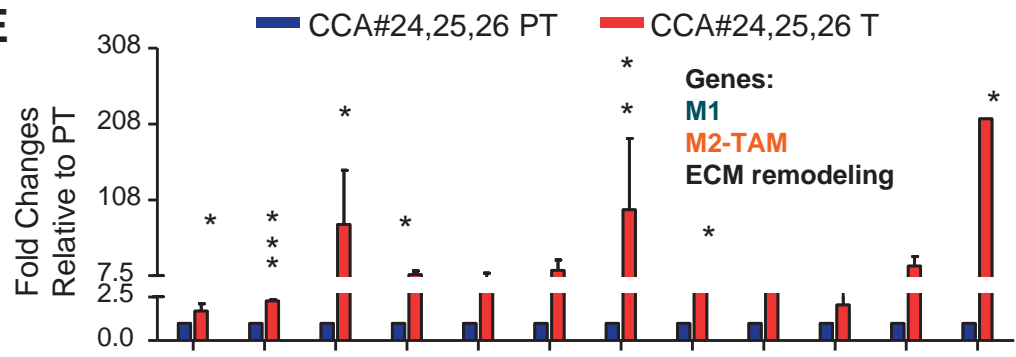

F

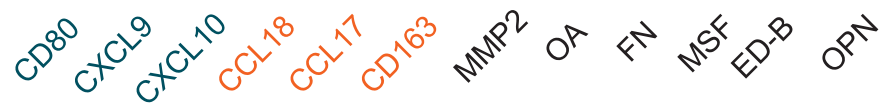
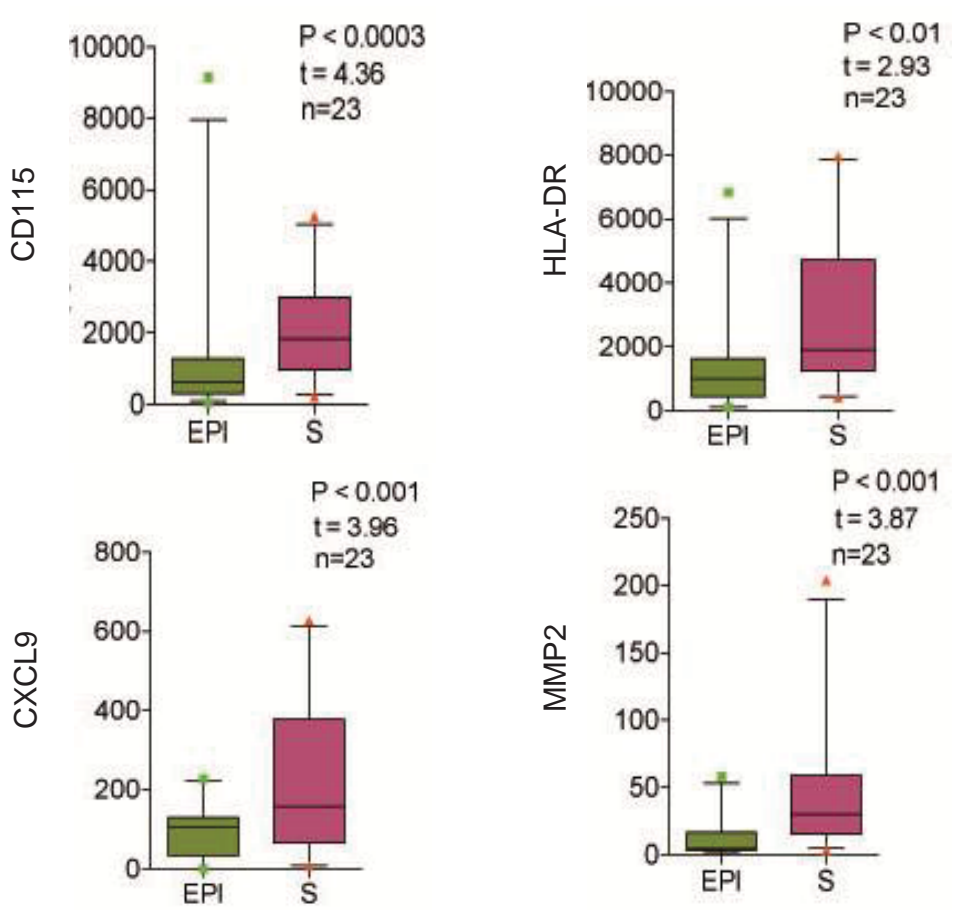

G
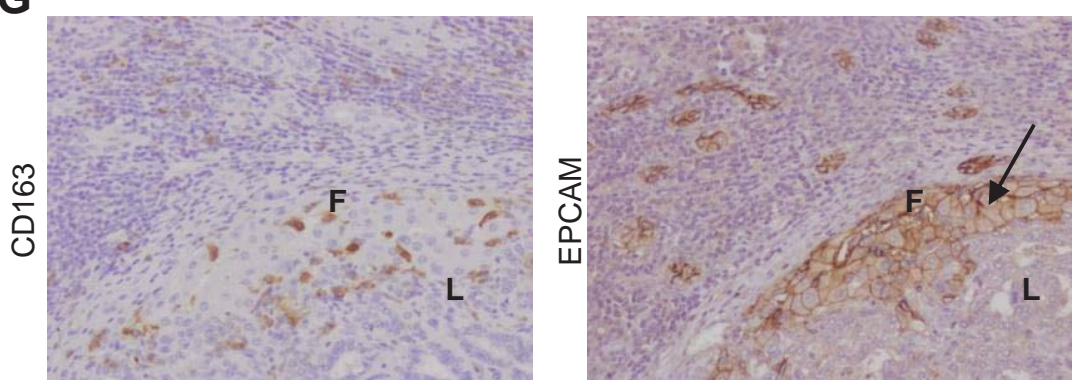

L

ֻู

岁

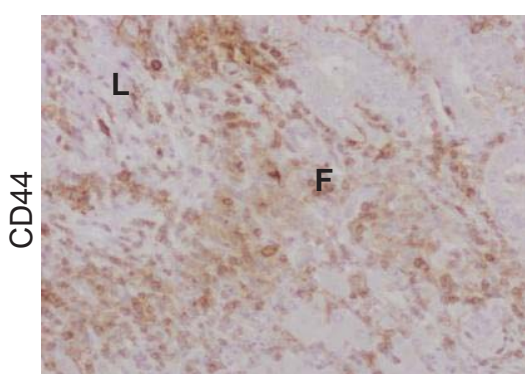


Fig. 6 A

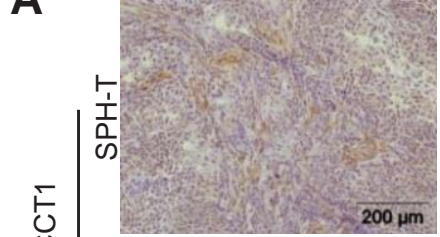

Uي

方

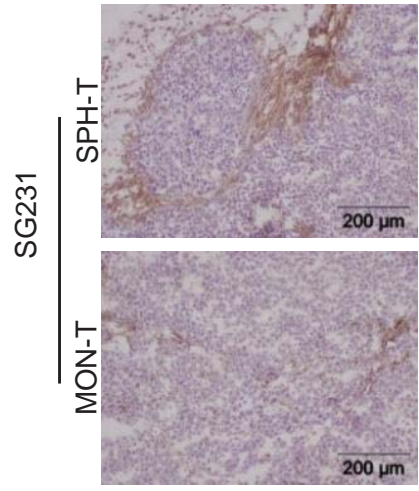

D

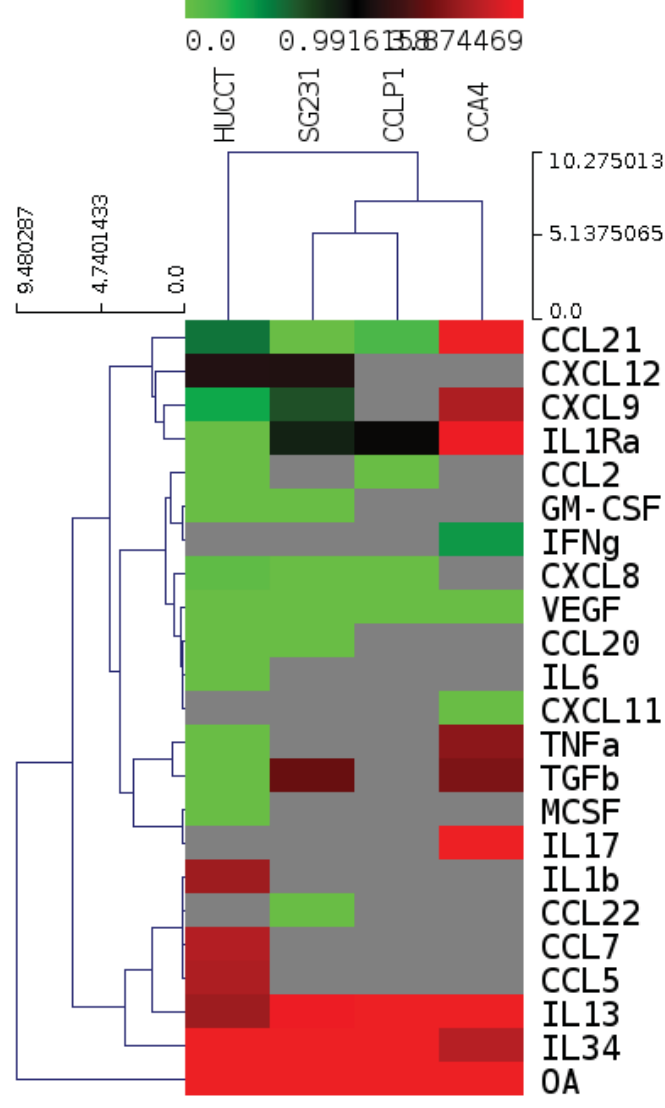

F

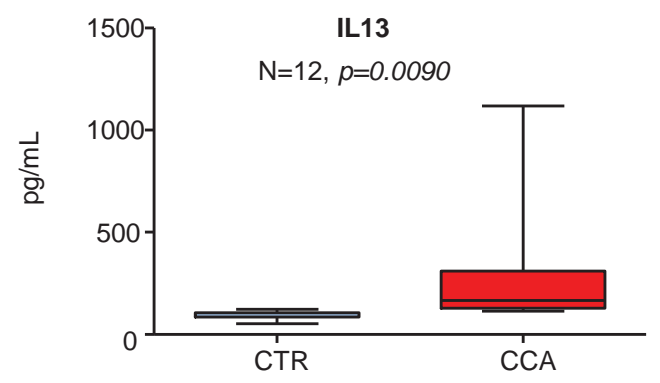

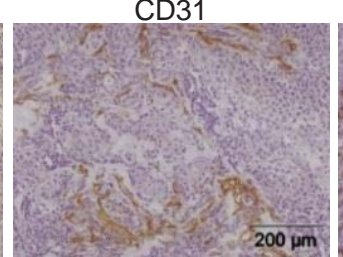
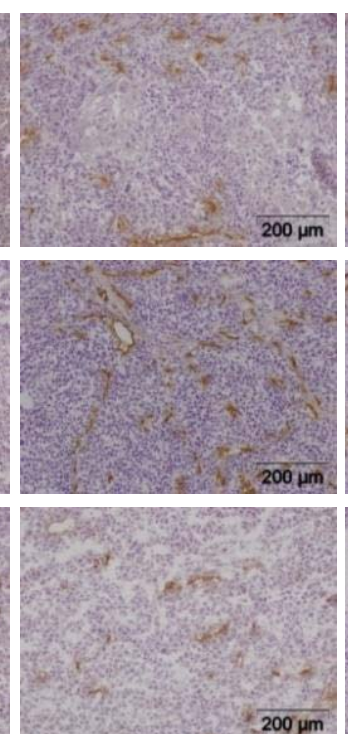

$200 \mu \mathrm{m}$

\section{E}

F4/80
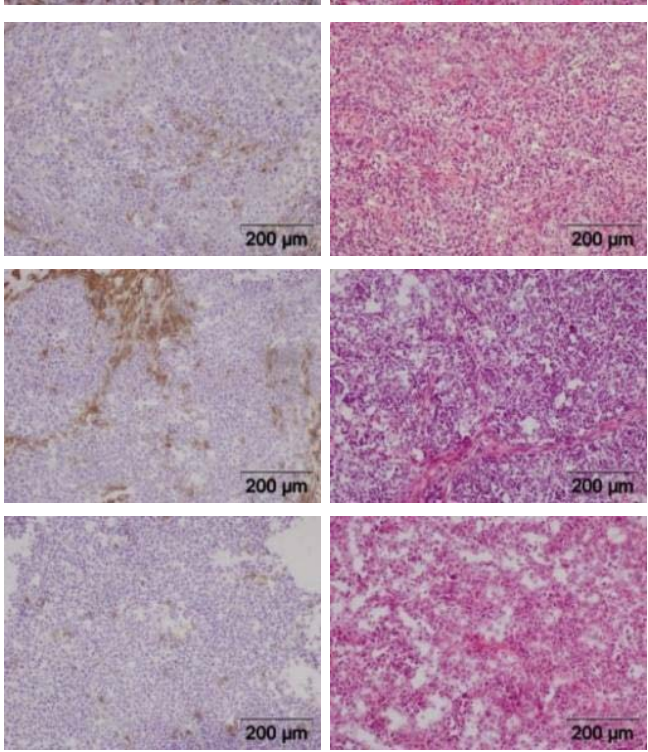

Sirius Red

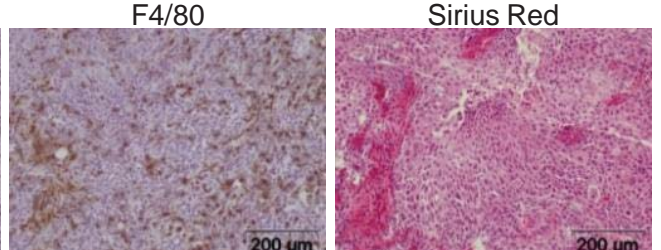

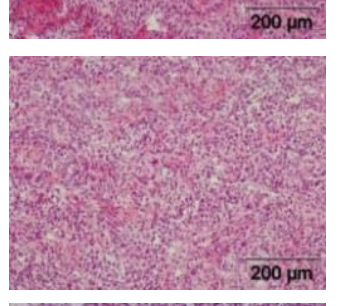

C

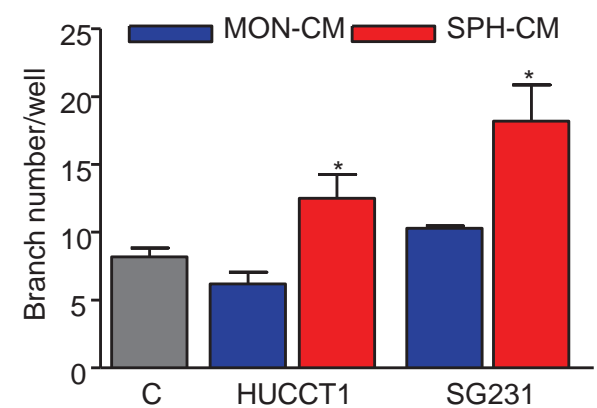

C
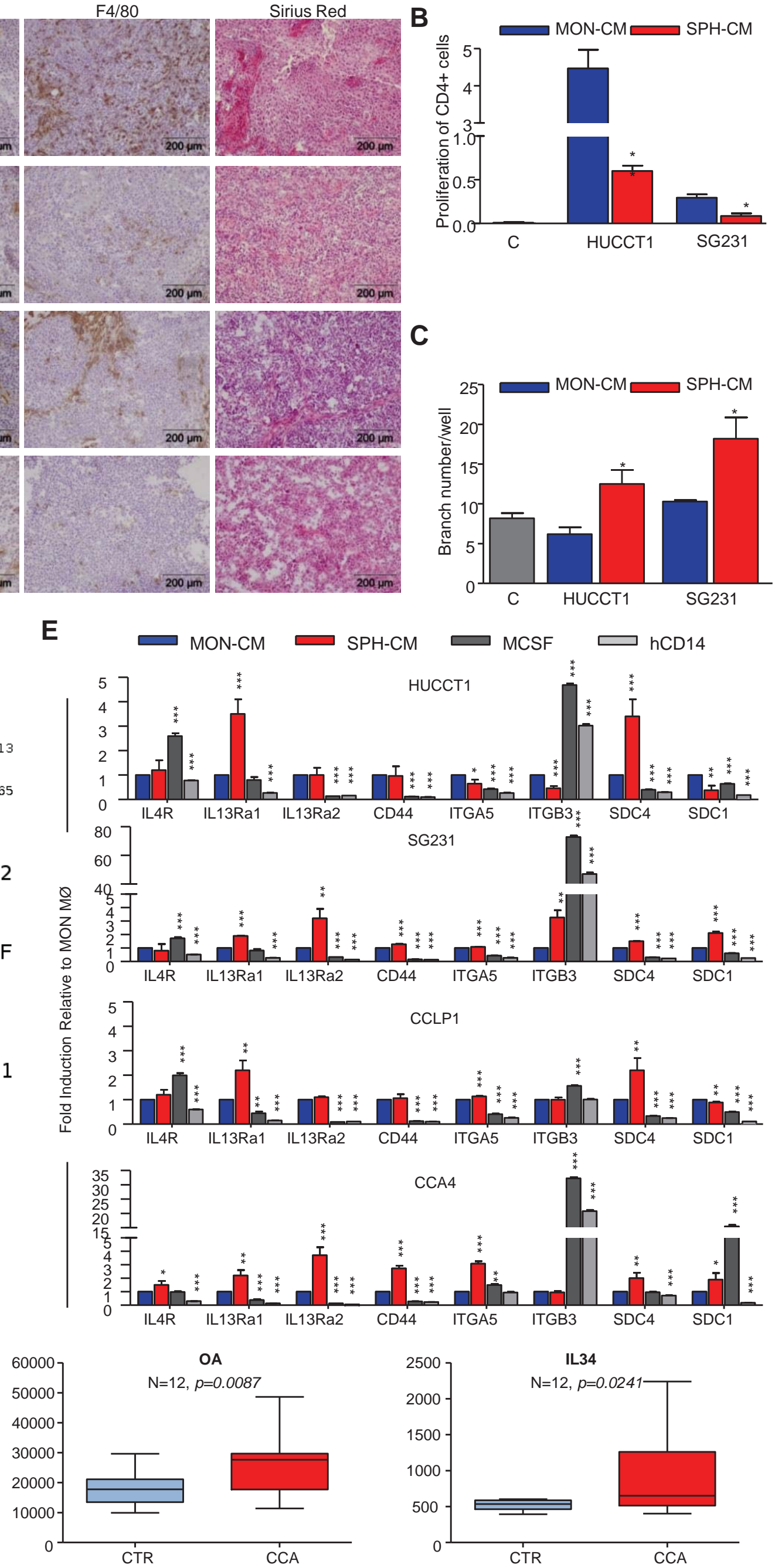
Fig. 7

A
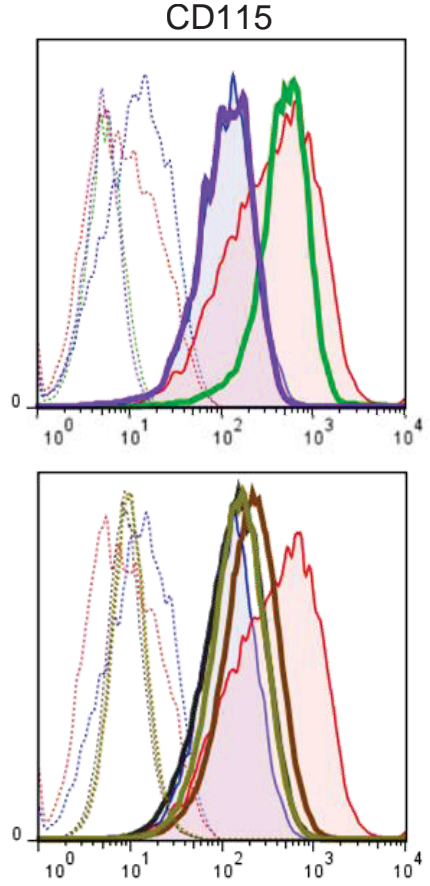
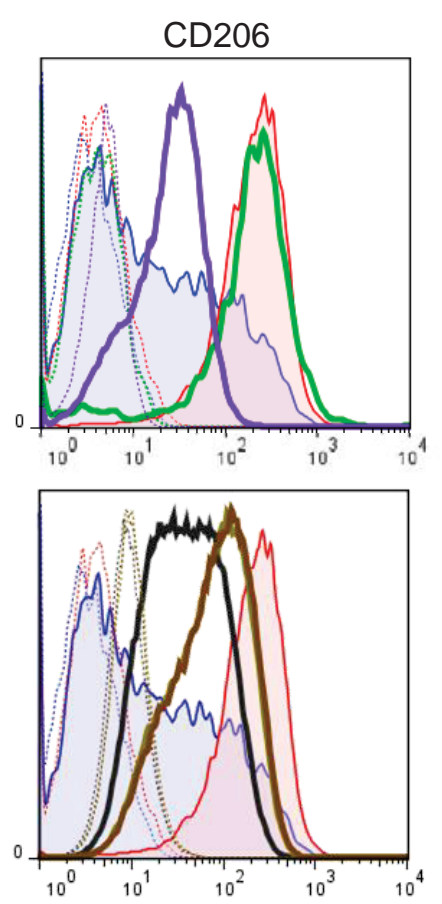

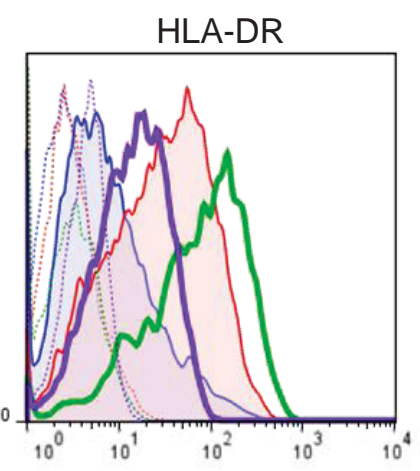

- MON-CM

- $\mathrm{SPH}-\mathrm{CM}$

-MON-CM(+IL13+OA+IL34)

- $\mathrm{SPH}-\mathrm{CM}$ (+Abs)

- SPH-CM (+Ab anti-IL13)

- SPH-CM (+Ab anti-OA)

- SPH-CM (+Ab anti-IL34)

B

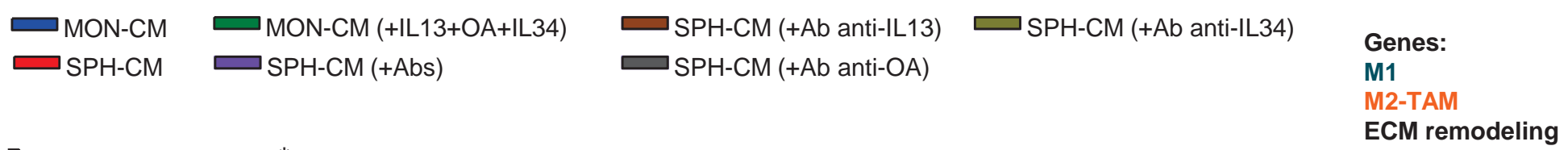

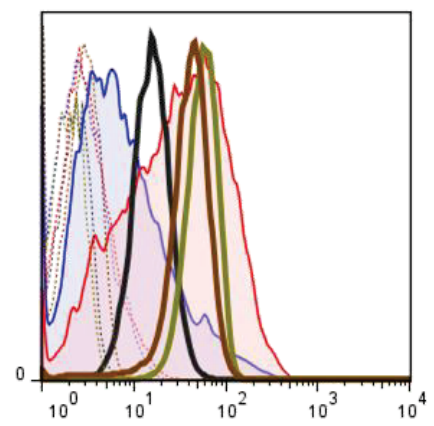

ECM remodeling

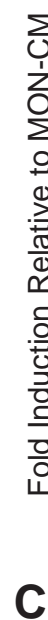

C

\section{o

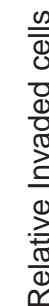

D

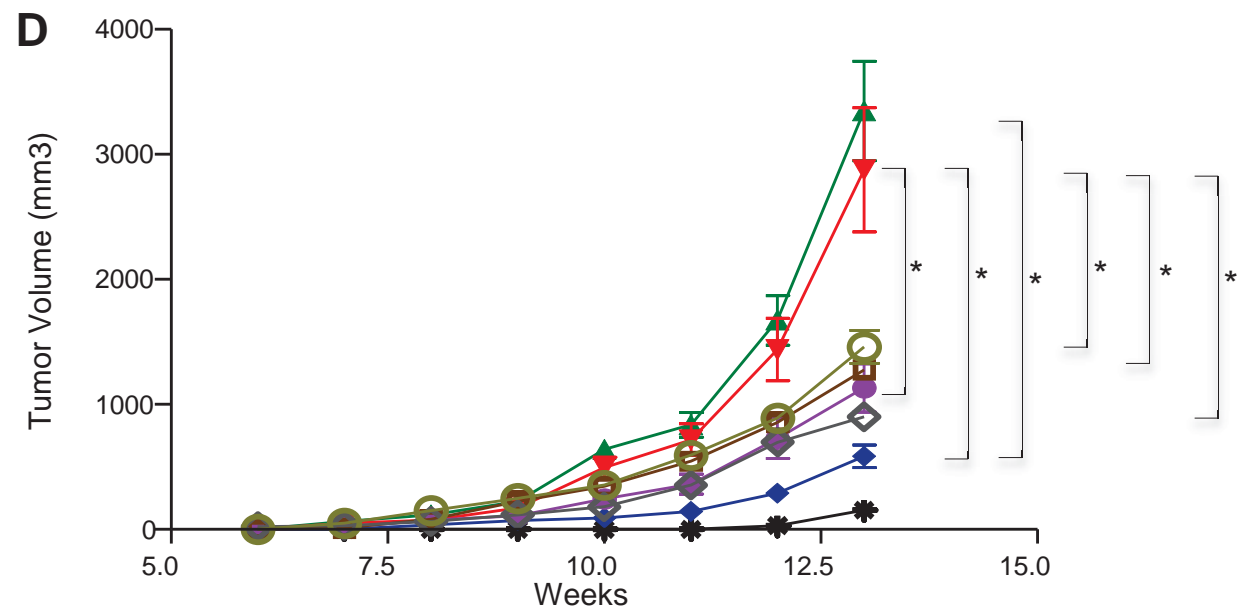

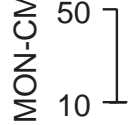

$7.5 \mathrm{~T}$
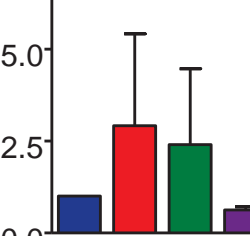

$\mathrm{c}^{80} \quad \mathrm{ct}^{\mathrm{CO}^{9}}$
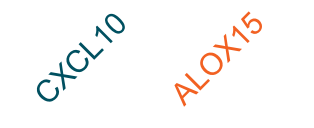

$0^{0^{18}}$

$o^{0^{\wedge}}$

$0^{\circ}$
$0^{10^{3}}$

or op
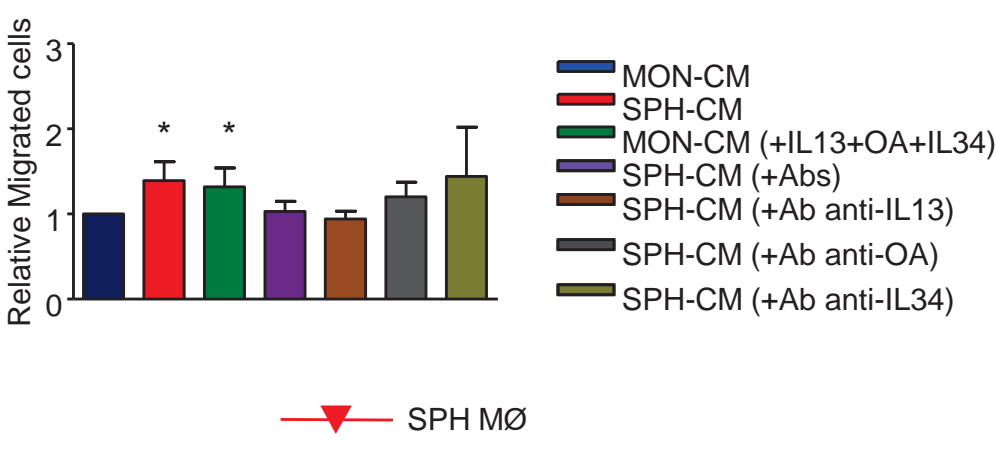

$\longrightarrow$ MON M $\varnothing$

$\longrightarrow$ MON (+IL13+OA+IL34) M

$-\mathrm{SPH}(+\mathrm{Abs}) \mathrm{M} \varnothing$

曰- SPH+Ab IL13

- $-\mathrm{SPH}+\mathrm{Ab}$ IL34

$\vartheta \mathrm{SPH}+\mathrm{Ab} O \mathrm{~A}$

— w/o M $\varnothing$ 
